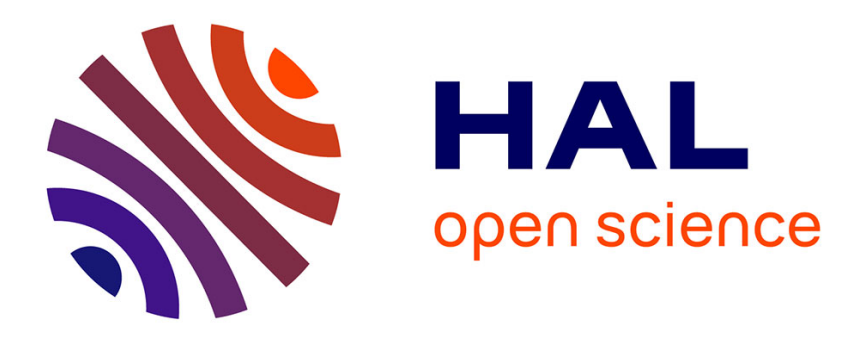

\title{
Large population and size scale limit of a stochastic particle model \\ Philippe Michel
}

\section{To cite this version:}

Philippe Michel. Large population and size scale limit of a stochastic particle model. Mathematical Models and Methods in Applied Sciences, 2017, 10.1142/S0218202517500105 . hal-01310264

\section{HAL Id: hal-01310264 \\ https://hal.science/hal-01310264}

Submitted on 2 May 2016

HAL is a multi-disciplinary open access archive for the deposit and dissemination of scientific research documents, whether they are published or not. The documents may come from teaching and research institutions in France or abroad, or from public or private research centers.
L'archive ouverte pluridisciplinaire HAL, est destinée au dépôt et à la diffusion de documents scientifiques de niveau recherche, publiés ou non, émanant des établissements d'enseignement et de recherche français ou étrangers, des laboratoires publics ou privés. 


\title{
Large population and size scale limit of a stochastic particle model describing an age and space-structured population
}

\author{
P. Michel*†
}

March 22, 2016

\begin{abstract}
The aim of this work is to study this stochastic individual-based model, structured with respect to age (progression within the cell cycle) and space (radial distance from the oocyte). We prove the existence of solutions and the convergence in large population and size scale limit to a partial differential equation.
\end{abstract}

Key-words: stochastic processes, partial differential equation, convergence.

AMS class. No: 35L60, 35L65, 60G55

\section{Contents}

1 Introduction $\quad 2$

2 Main results $\quad 5$

3 Proof of the main theorem $\quad 7$

3.1 Proof of theorem 2.2 Part I : Convergence of the stochastic process $Z_{t}^{M, \epsilon}$ as $M \rightarrow \infty \ldots \ldots$

3.2 Proof of theorem 2.2 Part II : Convergence of $\rho_{t}^{\epsilon}$ as $\epsilon \rightarrow 0 \ldots \ldots \ldots \ldots \ldots$

3.3 Proof of theorem 2.2 Part III : Convergence of $Z_{t}^{M, \epsilon}$ as $M \rightarrow \infty$ and $\epsilon \rightarrow 0 \ldots \ldots$

4 Conclusion $\quad 9$

5 Annex $r$

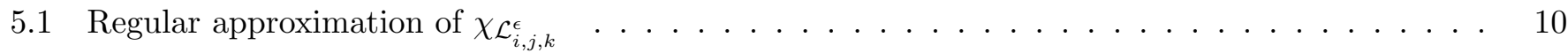

5.2 Proof of proposition 2.1 : Existence of the stochastic process $Z_{t}^{M, \epsilon} \ldots \ldots \ldots \ldots$

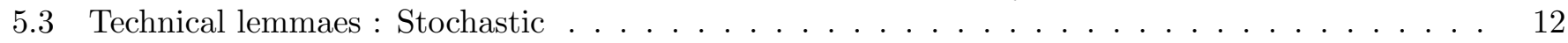

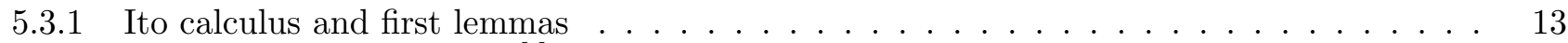

5.3 .2 Doob decomposition of $Z_{t}^{M, \epsilon} \ldots \ldots \ldots \ldots \ldots \ldots \ldots \ldots$

5.3 .3 Uniform bounds on $Z_{t}^{M, \epsilon} \ldots \ldots \ldots \ldots \ldots \ldots \ldots \ldots$

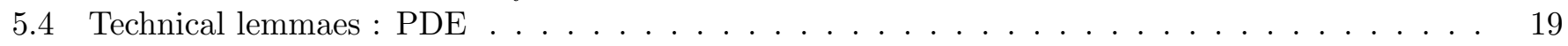

5.4.1 Proof of existence and uniqueness of solution to $(12) \ldots \ldots \ldots \ldots \ldots$

5.4 .2 Compactness of $M_{\epsilon}^{1}(t, p):=\int \rho^{\epsilon}(t, a, p) d a \ldots \ldots \ldots \ldots \ldots \ldots$

5.4 .3 Proof of existence and uniqueness of solution to (15)bis . . . . . . . . . . . . 24

${ }^{*}$ Université de Lyon, CNRS, Ecole Centrale de Lyon, Institut Camille Jordan, 69134 Ecully Cedex, France

${ }^{\dagger}$ INRIA - MYCENAE project team 


\section{Introduction}

The development of ovarian follicles is a unique instance of a morphogenesis process resulting from the interactions between somatic cells (granulosa cells) and germ cell (oocyte). In mammals, the initiation of follicular development from the pool of resting follicles is characterized by an increase in the oocyte size concomitant with the surrounding granulosa cells proliferating (see [19]). In [5], the authors have introduced a multi-scale stochastic model, taking in accounts for the molecular dialogue existing between the oocyte and granulosa cells, of the primordial follicle development. We propose, here, to study mathematically this model. The main line of this model can be summarized as follows :

i) there are a population of small cells of diameter $\epsilon$ proliferate around large cell of radius $r_{O}$ (see fig. 1),

ii) there is a dialog (depending on the distance) between the large cell and the small cells (see fig. 1) which links growth and proliferation.

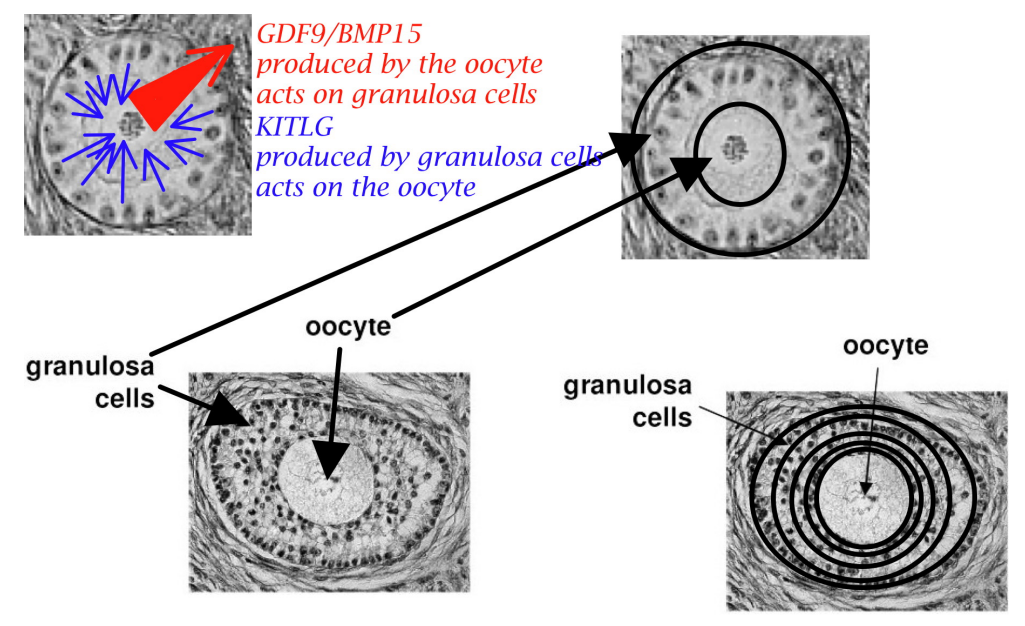

Figure 1: Development of preantral follicles. Each follicle is comprised of a germ cell (the oocyte) and granulosa cells. The oocyte produces GDF9/BMP15 which make granulas cells proliferate around the oocyte and granulosa cells (small ones) produce KITLG that make the oocyte grows. Therefore, the development of each follicle (in its basal follicular development), is coordinated by tight interactions existing between the oocytes and their surrounding granulosa cells.

The age of a cell is simply a positive real number and $A g e=\mathbb{R}_{+}$. To simplify the computation, we choose an equivalent formulation of this model : the large cell keeps a constant radius $(=1)$ but the local volume of space around the oocyte evolves with respect to time. The physical space is

$$
\text { Space }=\mathbb{R}^{3} / B(0,1), \quad \text { where } B(0,1)=\{(r, \theta, \phi) \in \text { Space }: r<1\} .
$$

Let $\epsilon>0$ the diameter of a granulosa cell, then $i^{t} h$ layer is given by

$$
\mathcal{L}_{i}^{\epsilon}=\{(r, \theta, \phi) \in \text { Space }: r \in[1+(i-1) \epsilon, 1+i \epsilon[\}
$$

and for $N=\mathbb{E}_{n t}(1 / \epsilon) \in \mathbb{N}^{*}$, a subdivision of these layers (see fig. 2) is given by

$$
\begin{aligned}
\mathcal{L}_{i, j, k}^{\epsilon}=\{(r, \theta, \phi) \in \text { Space }: r \in[1+(i-1) \epsilon, 1+i \epsilon[ & \\
& \theta \in\left[\pi \frac{(j-1)}{N}, \pi \frac{j}{N}\left[, \quad \phi \in\left[-\pi+2 \frac{k-1}{N} \pi,-\pi+2 \frac{k}{N} \pi[\} .\right.\right.\right.
\end{aligned}
$$




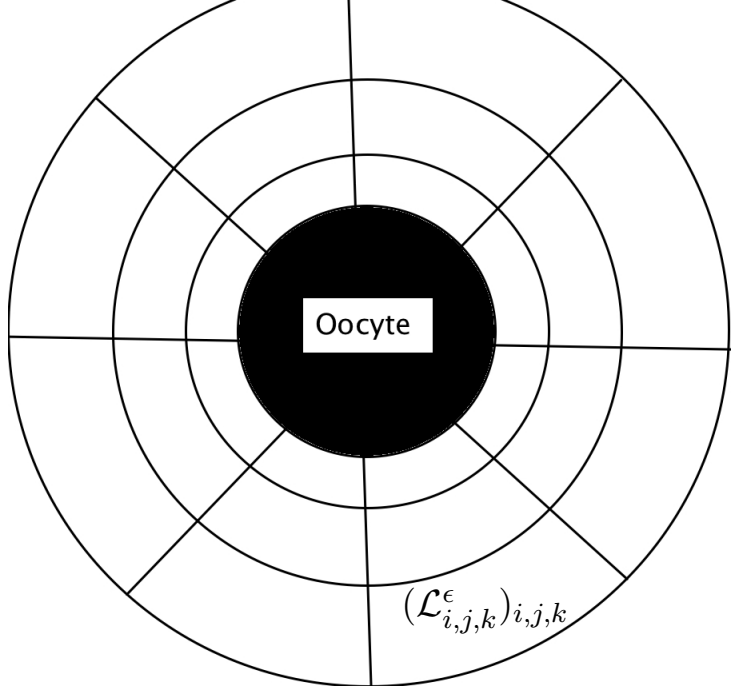

Figure 2: Space layer decomposition. The physical space surround the ball (oocyte) in its center. We subdivide this space in layers $\left(\mathcal{L}_{i}^{\epsilon}\right)_{i}$ and mesh these layers uniformly $\left(\mathcal{L}_{i, j, k}^{\epsilon}\right)_{i, j, k}$ of volumes $\left(V_{o l} l_{i, j, k}\right)_{i, j, k}$. by

For $\epsilon>0$, the volume of each $\mathcal{L}_{i}^{\epsilon}\left(\right.$ resp. $\mathcal{L}_{i, j, k}^{\epsilon}$ ) (which evolves with the radius of this oocyte $r_{O}$ ) are given

$$
V o l_{i}^{\epsilon}=\frac{4 \pi}{3}\left[\left(r_{O}+i \epsilon\right)^{3}-\left(r_{O}+(i-1) \epsilon\right)^{3}\right]=\frac{4 \pi}{3}\left[\epsilon^{3}\left(3 i^{2}-3 i+1\right)+3 \epsilon^{2} r_{O}(2 i-1)+3 \epsilon r_{O}^{2}\right],
$$

and

$$
\operatorname{Vol}_{i, j, k}^{\epsilon}=\operatorname{Vol}_{i}^{\epsilon} / N^{2} \sim \epsilon^{2} \frac{4 \pi}{3}\left[\epsilon^{3}\left(3 i^{2}-3 i+1\right)+3 \epsilon^{2} r_{O}(2 i-1)+3 \epsilon r_{O}^{2}\right] .
$$

For each time, a cell is defined by its age and its position, therefore by a dirac mass on Age $\times$ Space. The whole population is then characterized by a punctual measure on this set, i.e., in $\mathcal{M}_{P}($ Space $\times$ Age $)$. Let

$$
Z_{0}^{M, \epsilon}(d a, d p)=\frac{1}{M} \sum_{k=1}^{N_{0}^{M, \epsilon}} \delta_{\left(a_{k}^{M, \epsilon}, x_{k}^{M, \epsilon}\right)} \in \mathcal{M}_{P}(\text { Space } \times \text { Age }),
$$

where $M$ is a normalization parameter and $\left(a_{k}, x_{k}\right)_{k} \subset$ Age $\times$ Space, the initial population sequence, such that

$$
\sup _{M, \epsilon}\left(N_{0}^{M, \epsilon} / M\right)<\infty
$$

and $r_{O}^{M, \epsilon}(0)=r_{0}>0$. Let $Q\left(d s, \operatorname{Compt}(d n), d \Theta, \Pi_{s-}\left(p, d p^{\prime}\right)\right)$ be a Poisson point measure on $\mathbb{R}_{+} \times \varepsilon=$ $\mathbb{R}_{+} \times \mathbb{N} \times \mathbb{R}_{+} \times \mathbb{R}^{3}$ with intensity

$$
q\left(d s, \operatorname{Compt}(d n), d \Theta, \Pi_{s-}^{\epsilon}\left(p, d p^{\prime}\right)\right)=d s \otimes \operatorname{Compt}(d n) \otimes d \Theta \otimes \Pi_{s-}^{\epsilon}\left(p, d p^{\prime}\right), \quad d p^{\prime}=r^{2} \sin (\theta) d r d \theta d \phi,
$$

and independent of $Z_{0}^{M, \epsilon}$ (see $\left.[4,25,17,15,3]\right)$. Let us denote $X_{k}^{M, \epsilon}(t)$ and $A_{k}^{M, \epsilon}(t)$ the position and age of the $k^{\text {th }}$ individual at time $t$ (ranked in the lexicographic order on $\mathbb{R}^{3} \times \mathbb{R}_{+}$, see $[4,25,17,15,3]$ for details). Then the oocyte radius follows the equation

$$
r_{O}^{M, \epsilon}(t)=r_{O}^{M, \epsilon}(0)+\sum_{i, j, k} \kappa((i-1) \epsilon) \int_{0}^{t}\left(r_{O}^{M, \epsilon}(s-)\right)^{\alpha}\left\langle\Psi_{i, j, k}^{\epsilon}, Z_{s-}^{M, \epsilon}\right\rangle d s
$$

where $\alpha<0$ and $\kappa \in C_{b}^{0}\left(\mathbb{R}_{+}, \mathbb{R}_{+}\right)$and $\Psi_{i, j, k}^{\epsilon}$ a regular approximation of the characteristic function $\chi_{\mathcal{L}_{i, j, k}^{\epsilon}}$ (see (28) in the annex : section 5.1). The population at time $t$, denoted by $Z_{t}^{M, \epsilon}$, is the set of all individuals alive 
at time $t$ and follows the master equation

$$
\begin{aligned}
& Z_{t}^{M, \epsilon}(d a, d p)= \frac{1}{M} \sum_{k=1}^{N_{0}^{M, \epsilon}} \delta_{\left(A_{k}^{M, \epsilon}(0)+t, X_{k}^{M, \epsilon}(0)\right)} \\
&\left.+\frac{1}{M} \int_{0}^{t} \int_{\varepsilon} 1_{n<N_{s-}^{M, \epsilon}\left[\left(2 \delta_{\left(t-s, X_{n}^{M}\right.}^{M, \epsilon}(s-)\right)\right.}-\delta_{\left(A_{n}^{M, \epsilon}(s-)+t-s, X_{n}^{M, \epsilon}(s-)\right)}\right) 1_{0 \leq \Theta<B_{n, s-}^{M, \epsilon}} \\
&\left.+\left(\delta_{\left(A_{n}^{M, \epsilon}(s-)+t-s, p^{\prime}\right)}-\delta_{\left(A_{n}^{M, \epsilon}(s-)+t-s, X_{n}^{M, \epsilon}(s-)\right)}\right) 1_{0 \leq \Theta-B_{n, s-}^{M, \epsilon}<P_{s-}^{\epsilon}\left(X_{n}^{M, \epsilon}(s-)\right)}\right] Q\left(d s,(d n), d \Theta, \Pi_{s-}^{\epsilon}\left(p, d p^{\prime}\right)\right),
\end{aligned}
$$

where

$$
\begin{gathered}
B_{n, s-}^{M, \epsilon}=B\left(A_{n}^{M, \epsilon}(s-), X_{n}^{M, \epsilon}(s-)\right), \text { with } B(a, p)=1-e^{-a / \lambda\left(\|p\|_{2}\right)}, \quad \lambda \in C^{0}\left(\mathbb{R}_{+}, \mathbb{R}_{+}\right), \\
P_{s-}^{\epsilon}(p)=C^{\epsilon} \sum_{i, j, k} \Psi_{i, j, k}^{\epsilon}(p) R\left(\left\langle\Psi_{i, j, k}^{\epsilon}, Z_{s-}^{M, \epsilon}\right\rangle \frac{V o l_{G}}{V o l_{i, j, k}^{\epsilon}}\right), \text { with } R(x)=\frac{1}{1+e^{-\frac{x-\mu}{\sigma}}},
\end{gathered}
$$

and

$$
\Pi_{s-}^{\epsilon}\left(p, d p^{\prime}\right)=\frac{\Psi^{\epsilon}\left(\left|p^{\prime}-p\right|\right) G_{s-}^{\epsilon}\left(p^{\prime}\right) d p^{\prime}}{\iiint \Psi^{\epsilon}(|q-p|) G_{s-}^{\epsilon}(q) d q}, \text { with } G_{s-}^{\epsilon}\left(p^{\prime}\right)=1-\frac{P_{s-}^{\epsilon}\left(p^{\prime}\right)}{C^{\epsilon}},
$$

where $C^{\epsilon}=C / \epsilon^{2}>0$.

The main objective of the paper is to prove the convergence of the stochastic processes $\left(Z_{t}^{M, \epsilon}(d a, d p)\right)_{\epsilon, M}$ as the population increases to infinity and the size of a cell converges to zero (the existence of solutions to the system (7)-(17) is quite classical (see $[4,25,17,15,3]$ ) and so be proved in annex 5). Difficulties of this work appear in the manipulation of stochastic and deterministic tools. Indeed, stochastic processes, their convergence or/and tightness (compactness) are powerfull mathematical tools but are not well suited for the punctual (or regular) convergence, therefore for a fixed initial population size (in an equivalent way, fixing $M$ ) we cannot pass to the limit as $\epsilon$ converges to zero. Thus, its not completly clear that $\left(Z_{t}^{M, \epsilon}(d a, d p)\right)_{\epsilon, M}$ converge for all sequences of $\left(\epsilon_{k}, M_{k}\right) \rightarrow(0, \infty)$. We show that the limit (étroite-weak limit [15,4] for similar studies) of $\left(Z_{t}^{M, \epsilon}(d a, d p)\right)_{\epsilon, M}$ (for a fixed $\epsilon$ and $M \rightarrow \infty$ ) is well defined (unique) and is a strong solution to the following partial differential equation

$$
\text { Intermediate eq. }\left\{\begin{array}{l}
\left(\frac{\partial}{\partial t}+\frac{\partial}{\partial a}\right) \rho^{\epsilon}-\iiint P_{\infty}^{\epsilon}\left(p^{\prime}\right) \frac{\Pi_{\infty}^{\epsilon}\left(p^{\prime}, d p\right)}{d p} \rho^{\epsilon}\left(t, a, p^{\prime}\right) d p^{\prime}+P_{\infty}^{\epsilon}(p) \rho^{\epsilon}(t, a, p)=0, \\
r_{O}^{\epsilon}(t)=r_{O}^{\epsilon}(0)+\sum_{i, j, k} \kappa((i-1) \epsilon) \int_{0}^{t}\left(r_{O}^{\epsilon}(s)\right)^{\alpha} \iiint \int \Psi_{i, j, k}^{\epsilon}(p) \rho^{\epsilon}(t, a, p) d a d p d s \\
\left.\rho^{\epsilon}(t, a, p)\right|_{a=0}=2 \int B(a, p) \rho^{\epsilon}(t, a, p) d a d p, \quad \rho^{\epsilon}\left(0, a, p^{\prime}\right)=\rho_{0}^{\epsilon}(a, p), \quad r_{O}^{\epsilon}(0)=r_{0} .
\end{array}\right.
$$

with $M_{\epsilon}^{1}(t, p):=\int \rho^{\epsilon}(t, a, p) d a$,

$$
\begin{gathered}
P_{\infty}^{\epsilon}\left(p^{\prime}\right)=\frac{C}{\epsilon^{2}} \sum_{i, j, k} \Psi_{i, j, k}^{\epsilon}\left(p^{\prime}\right) R\left(\frac{\iiint \Psi_{i, j, k}^{\epsilon}(p) M_{\epsilon}^{1}(t, p) d p V o l_{G}}{V o l_{i, j, k}^{\epsilon}}\right) \text {, with } R(x)=\frac{1}{1+e^{-\frac{x-\mu}{\sigma}}}, \\
\Pi_{\infty}^{\epsilon}\left(p^{\prime}, d p\right)=\frac{\Psi^{\epsilon}\left(\left|p^{\prime}-p\right|\right) G_{s-}^{\epsilon}(p) d p}{\iiint \Psi^{\epsilon}\left(\left|q-p^{\prime}\right|\right) G_{s-}^{\epsilon}(q) d q}, \text { with } G_{s-}^{\epsilon}(.)=1-\sum_{i, j, k} \Psi_{i, j, k}^{\epsilon}(.) R\left(\frac{\iiint \Psi_{i, j, k}^{\epsilon}(p) M_{\epsilon}^{1}(t, p) d p V o l_{G}}{V o l_{i, j, k}^{\epsilon}}\right) .
\end{gathered}
$$

Then, we prove that, the limit as $\epsilon$ converge to zero, is a weak solution (using Sobolev spaces and weak-* convergence [2]) to the following nonlinear partial differential equation (Transport [20, 22], Keller Segel type $[12,13])$ 


$$
\text { Final eq. }\left\{\begin{array}{l}
\left(\frac{\partial}{\partial t}+\frac{\partial}{\partial a}\right) \rho+\operatorname{div}(C \rho R \nabla(\log (1-R)))=0, \\
\left.\rho\right|_{a=0}=2 \int B(a, p) \rho(t, a, p) d a, \quad \rho_{t=0}=\rho_{0}, \\
r_{O}(t)=r_{O}(0)+\int_{0}^{t}\left(r_{O}^{\epsilon}(s)\right)^{\alpha} \iiint \int \kappa(r) \rho^{\epsilon}(t, a, p) d a d p d s, \quad r_{O}(0)=r_{0} .
\end{array}\right.
$$

with $C>0, R=R\left(\int_{\mathbb{R}_{+}} \rho(t, a, p) d a \frac{V o l_{G}\left(3 r^{2}+6 r+3\right)}{3 r^{2}+6 r r_{O}(t)+3 r_{O}(t)^{2}}\right)$ and

$$
r_{O}^{\prime}(t)=\left(r_{O}(t)\right)^{\alpha} \iiint \int_{\text {Age } \times \text { Space }} \kappa(r) \rho(t, a, p) r^{2} \sin (\theta) d a d r d \theta d \phi, \quad r_{O}(0)=r_{0} .
$$

Therefore, the main difficulty is to mix both approach to prove the convergence. In section 2 , we give main theorems of convergence and proof is done in section 3 (we put in annex (section 5) the most technicals lemmaes).

\section{Main results}

Before proving the convergence of the stochastic process $Z_{t}^{M, \epsilon}$, we first show that we have existence for all $M$ and $\epsilon$ of $Z_{t}^{M, \epsilon}$ (proposition 2.1). Then, we adopt the following approach (see fig. 2.2) : we show, in theorem 2.2 I, that we can extract a subsequence $M_{k} \rightarrow \infty$ such that $\left(Z_{t}^{M_{k}, \epsilon}\right)_{M_{k}, \epsilon}$ converge to a solution $\rho^{\epsilon}$ to an intermediate partial differential equation (12), then we prove, in theorem 2.2 II, that we can extract a subsequence $\epsilon_{k} \rightarrow \infty$ such that $\rho^{\epsilon_{k}}$ converge to $\rho$ solution to (15). Finally, in theorem 2.2 III, we show that there exists a subsequence $\left(\epsilon_{k}, M_{k}\right) \rightarrow(0, \infty)$ such that $Z_{t}^{M, \epsilon}$ converges to $\rho$ (see fig. 2.2).

\section{Assumptions}

I- Geometric : Assume that for all $p$, we can define the matrix

$$
M_{\Psi}:=\frac{\iiint_{q} \Psi^{\epsilon}(|q-p|)(q-p)^{t}(q-p) d q}{\epsilon^{2} \iiint \Psi^{\epsilon}(|q-p|) d q},
$$

which satisfies

$$
\left\{\begin{array}{l}
M_{\Psi} \text { is definite positive } \\
\forall p \forall \epsilon, \quad 0<\inf _{\epsilon, p} \min \left\{\lambda \in S p\left(M_{\Psi}\right)\right\} \leq \sup _{\epsilon, p} \max \left\{\lambda \in S p\left(M_{\Psi}\right)\right\}<\infty \\
C M_{\Psi} \rightarrow{ }^{C^{0}(\text { Space })} C h(p) .
\end{array}\right.
$$

Remark 1 The construction we give in section 5.1 satisfies (17) and by symmetry of $\Psi^{\epsilon}$ we have directly that $C h(p)=C I d$ for all $p \in$ Space. Moreover, we notice that

$$
\operatorname{Vol}_{G} \iiint \Psi_{i, j, k}^{\epsilon} d p / \operatorname{Vol}_{i, j, k}^{\epsilon} \rightarrow^{\epsilon \rightarrow 0} \frac{\operatorname{Vol}_{G}\left(3 r^{2}+6 r+3\right)}{3 r^{2}+6 r r_{O}(t)+3 r_{O}(t)^{2}}
$$

II- Uniform bounds on $Z_{0}^{M, \epsilon}$ and $\rho_{0}^{\epsilon}(a, p)$ : assume there exists $m \geq 1$ and $w>0$ s.t.

$$
\sup _{(M, \epsilon) \in U_{w}} \mathbb{E}\left(\left(\iiint \int\left(1+a^{m}+r^{m}\right) Z_{0}^{M, \epsilon}(d a, d p)\right)^{2}+\left(\iiint \int\left(1+a^{2 m}+r^{2 m}\right) Z_{0}^{M, \epsilon}(d a, d p)\right)\right)<\infty
$$

with

$$
\begin{gathered}
U_{w}:=\{M \epsilon>w\} \\
\sup _{\epsilon} \int(1+\|p\|)\left[\int \rho_{0}^{\epsilon}(a, p) d a+\left(\int \rho_{0}^{\epsilon}(a, p) d a\right)^{2}+\left(\int\left|\frac{\partial}{\partial a} \rho_{0}^{\epsilon}(a, p)\right| d a\right)^{2}\right] d p<\infty
\end{gathered}
$$


II- Convergence of $Z_{0}^{M, \epsilon}$ and $\rho_{0}^{\epsilon}(a, p)$ :

$$
\begin{aligned}
& Z_{0}^{M, \epsilon}(d a, d p) \rightarrow_{M \rightarrow \infty} \rho_{0}^{\epsilon}(a, p) d a d p, \quad \text { with } \quad \rho_{0}^{\epsilon}(a, p) \in C_{0}^{1}\left(\mathbb{R}^{+} \times \mathbb{R}^{+} \times \mathbb{R}^{3}\right) \\
& \rho_{0}^{\epsilon}(a, p) \rightarrow{ }_{\epsilon \rightarrow 0}^{L^{2}\left(\mathbb{R}^{+} \times\right)(\text {Age } \times \text { Sapce })} \rho_{0}(a, p), \quad \text { with } \quad \rho_{0}(a, p) \in C_{0}^{1}\left(\mathbb{R}^{+} \times \mathbb{R}^{+} \times \mathbb{R}^{3}\right)
\end{aligned}
$$

Proposition 2.1 (Existence) Under assumptions (6)-(11) and (18) there exists a solution $Z_{t}^{M, \epsilon} \in D\left(\mathbb{R}_{+}, \mathcal{M}_{P}\right)$ and $r_{O} \in C^{0} \cap C_{m}^{1}\left(\mathbb{R}_{+}\right)$to (7)-(8) for all $M, \epsilon>0$. Moreover, assuming that (18) is satisfied then we have that

$$
\sup _{M, \epsilon} \mathbb{E}\left(\sup _{t \in[0, T]}\left(\iiint \int\left(1+a^{m}+r^{m}\right) Z_{t}^{M, \epsilon}(d a, d p)\right)^{2}+\left(\iiint \int\left(1+a^{2 m}+r^{2 m}\right) Z_{t}^{M, \epsilon}(d a, d p)\right)\right)<\infty, \quad \forall T>0 .
$$

The proof is given in section 5.2.

Theorem 2.2 We have that.

I) Assume that (21) and (18) are satisfied. Then, for all $\epsilon>0,\left(Z_{t}^{M, \epsilon}(d a, d p)\right)_{M}$ is tight on $\mathbb{D}\left(\mathbb{R}_{+},\left(\mathcal{M}_{F}(A g e \times\right.\right.$ Space), weak)). Its limit values $\lim _{M_{k} \rightarrow \infty} Z_{t}^{M_{k}, \epsilon}(d a, d p)=\rho^{\epsilon}(t, a, p)$ dadp, are continuous measure-valued process satisfying (12)-(14).

II) Assume that (22), (20) and (17) are satisfied then $\left(\rho^{\epsilon}, r_{O}^{\epsilon}\right)$ solution to (12) weakly converge to $\left(\rho, r_{O}\right)$ weak solution to (15)-(16).

III) Assume that (21), (22), (18) and (17) are satisfied. Then, for all $C>0$, there exists a subsequence $\left(\epsilon_{k}, M_{k}\right) \subset U_{C}$ s.t. $M_{k} \epsilon_{k} \rightarrow \infty$ and

$$
\lim _{k \rightarrow \infty}\left\langle Z_{t}^{M_{k}, \epsilon_{k}}(d a, d p), \psi\right\rangle=\langle\rho(t, a, p) d a d p, \psi\rangle, \quad \forall \psi \in C_{0}^{1},
$$

weak solution to (15)-(16).

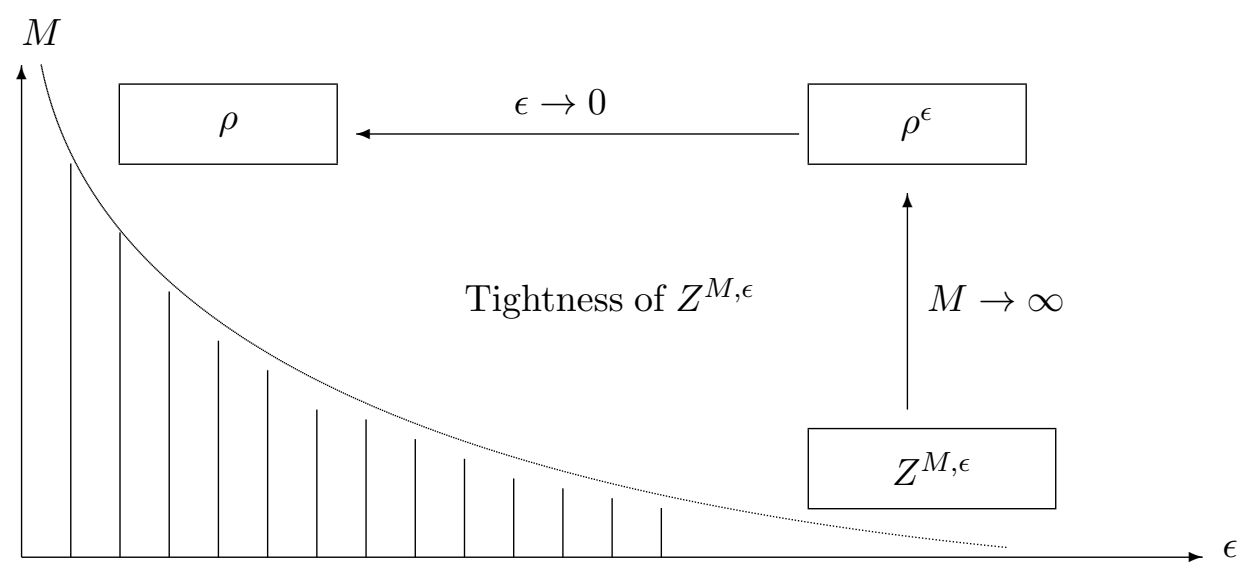

Figure 3: Convergence proof. 


\section{Proof of the main theorem}

The proof of the main theorem follows the scheme given in figure 2.2. The first and third part are stochastic processes results while the second point is a partial differential equation result.

\subsection{Proof of theorem 2.2 Part I : Convergence of the stochastic process $Z_{t}^{M, \epsilon}$ as $M \rightarrow \infty$}

To prove tightness of the sequence $Z_{t}^{M, \epsilon}(d a, d p)$ (as probability measure on $\mathbb{D}\left(\mathbb{R}_{+},\left(\mathcal{M}_{F}(\right.\right.$ Age $\times$ Space $)$, vague $\left.)\right)$ ), we use a Rolley criterium (see $[24,8,9]$ ) which establishes that it suffices to prove that for all $f$ of a dense subspace of $\left(C_{0}(\right.$ Age $\times$ Space, $\left.\mathbb{R}),\|\cdot\|_{\infty}\right)$ (here $C_{0}^{1}($ Age $\times$ Space, $\left.\mathbb{R})\right)$ the sequence $\left\langle f_{t}, Z_{t}^{M, \epsilon}\right\rangle$ is tight in $\mathbb{D}\left(\mathbb{R}_{+}, \mathbb{R}\right)$. In the section 5.3.2, we prove that

$$
\left\langle f_{t}, Z_{t}^{M, \epsilon}\right\rangle=\underbrace{\mathcal{M}_{t}^{M, \epsilon}(f)}_{\text {Martingale }}+\underbrace{\mathcal{V}_{t}^{M, \epsilon}(f)}_{\text {Finite Variation }} .
$$

Therefore, using a criterium of Aldous-Rebolledo [1, 9, 23], it suffices to prove that :

- for all $t \in \mathcal{T}$ (dense in $\left.\mathbb{R}_{+}\right),\left\langle\mathcal{M}_{t}^{M, \epsilon}(f)\right\rangle$ and $\mathcal{V}_{t}^{M, \epsilon}(f)$ are tight on $\mathbb{R}$

- for all $T>0, u>0, \eta>0$, there exists $\delta>0$ and $N_{0}^{M, \epsilon} \in \mathbb{N}$ s.t.

$$
\begin{gathered}
\sup _{M \geq N_{0}^{M, \epsilon}} \mathbb{P}\left(\left|\left\langle\mathcal{M}_{T_{M}}^{M, \epsilon}(f)\right\rangle-\left\langle\mathcal{M}_{S_{M}}^{M, \epsilon}(f)\right\rangle\right| \geq \eta, \quad T_{M}<S_{M}+\delta\right) \leq u, \\
\sup _{M \geq N_{0}^{M, \epsilon}} \mathbb{P}\left(\left|\mathcal{V}_{T_{M}}^{M, \epsilon}(f)-\mathcal{V}_{S_{M}}^{M, \epsilon}(f)\right| \geq \eta, \quad T_{M, \epsilon}<S_{M}+\delta\right) \leq u,
\end{gathered}
$$

for any sequences of stopping times $\left(S_{M}, T_{M}\right)$ of the natural filtration $\mathcal{F}_{M}$, so that $S_{M} \leq T_{M} \leq T$. Both points are a direct consequence of the bounds (42)-(44) (see [26] for more details). Using Prohorov theorem, we can extract a subsequence $Z_{t}^{M_{k}, \epsilon}(d a, d p)$ which vague converges to $Z_{t}^{\epsilon}(d a, d p)$ and by construction

$$
\sup _{t \in \mathbb{R}_{+}, f \in C^{1}(\text { Age } \times \text { Space })}\left|\left\langle f_{t}, Z_{t}^{M_{k}, \epsilon}\right\rangle-\left\langle f_{t}, Z_{t-}^{M_{k}, \epsilon}\right\rangle\right| \leq C s t \frac{\|f\|_{W^{1, \infty}}}{M_{k}}
$$

the limit process is a.e. continuous. Finally, using a result of convergence [16], to prove the weak convergence, it suffices to add tightness of $\left\langle 1, Z_{t}^{M, \epsilon}\right\rangle$ (which came directly from bound (38)). Now using (42), we have that the martingale part of the process satisfies

$$
\mathbb{E}\left(\left|\mathcal{M}_{t}^{M_{k}, \epsilon}(f)\right|\right)^{2} \leq \mathbb{E}\left(\left|\mathcal{M}_{t}^{M_{k}, \epsilon}(f)\right|^{2}\right)=\mathbb{E}\left(\left\langle\mathcal{M}_{t}^{M_{k}, \epsilon}(f)\right\rangle\right) \leq C s t \frac{\|f\|_{W^{1, \infty}}}{M_{k}} \rightarrow_{k \rightarrow \infty} 0 .
$$

By passing to the limit in (13)-(14), we have that

$$
P_{s-}^{\epsilon} \rightarrow_{M \rightarrow \infty} P_{\infty}^{\epsilon}(s-)=\frac{C}{\epsilon^{2}} \sum_{i, j, k} \Psi_{i, j, k}^{\epsilon}(.) R\left(\frac{\left\langle\Psi_{i, j, k}^{\epsilon}, Z_{s-}^{\epsilon}(d a, d p)\right\rangle V o l_{G}}{V o l_{i, j, k}^{\epsilon}}\right) \text {, with } R(x)=\frac{1}{1+e^{-\frac{x-\mu}{\sigma}}},
$$

and

$\Pi_{s-}^{\epsilon}(., d q) \rightarrow_{M \rightarrow \infty} \Pi_{\infty}=\frac{\Psi^{\epsilon}\left(\left|p^{\prime}-p\right|\right) G_{s-}^{\epsilon}\left(p^{\prime}\right) d p^{\prime}}{\iiint \Psi^{\epsilon}(|q-p|) G_{s-}^{\epsilon}(q) d q}$, with $G_{s-}^{\epsilon}\left(p^{\prime}\right)=1-\sum_{i, j, k} \Psi_{i, j, k}^{\epsilon}() R.\left(\frac{\left\langle\Psi_{i, j, k}^{\epsilon}, Z_{t}^{\epsilon}(d a, d p)\right\rangle V o l_{G}}{V_{o l}^{\epsilon} l_{i, j, k}}\right)$.

Therefore, we find that for all $f \in W^{1 \infty}$ (see lemma 5.2 and the section 5.3.2),

$$
\begin{aligned}
0=\left\langle f_{t}, Z_{t}^{M, \epsilon}\right\rangle-\left\langle f_{0}, Z_{0}^{M, \epsilon}\right\rangle-\int_{0}^{t}\left\langle\left(\frac{\partial}{\partial u}+\frac{\partial}{\partial a}\right) f(u, a, p), Z_{u}^{M, \epsilon}(d a, d p)\right\rangle d u \\
-\int_{0}^{t}\left\langle(2 f(s, 0, p)-f(s, a, p)) B(a, p)+\int_{\text {Space }}\left(f\left(s, p, p^{\prime}\right)-f(s, a, p)\right) P_{\infty}^{\epsilon}(s-)(p) \Pi_{\infty}\left(p, d p^{\prime}\right), Z_{s}^{M, \epsilon}\right\rangle d s
\end{aligned}
$$


is satisfied. The limit is a weak solution to the partial differential equation (12), which is unique (see the proof of II ).

\subsection{Proof of theorem 2.2 Part II : Convergence of $\rho_{t}^{\epsilon}$ as $\epsilon \rightarrow 0$}

Using a fixed point theorem (contraction in Banach space), we prove (in lemma 5.8) the existence of solution to the master equation (12). To prove the convergence as $\epsilon \rightarrow 0$, we first prove, in lemmaes 5.9-5.12, that under assumption (20), for any $T>0, M_{\epsilon}^{1}(t, p):=\int \rho^{\epsilon}(t, a, p) d a$ (resp. $\rho^{\epsilon}$ ) belongs to a compact set of $L^{2}([0, T] \times$ Space $)\left(\right.$ resp. $L^{2}([0, T] \times$ Age $\times$ Space $\left.)\right)$ and $\nabla M_{\epsilon}^{1}(t, p)$ belongs to a weak-* compact set of $L^{2}([0, T] \times$ Space $)$.

Assume (22) - (17), let $f \in C_{c}^{1}($ Age $\times$ Space), multiply (12) by $f$ and integrate with respect to $a$ : we find that

$$
\begin{aligned}
\frac{\partial}{\partial t} \int_{p} f(a, p) \rho^{\epsilon}(t, a, p) d a d p-\iint B(a, p) f(a, p) \rho^{\epsilon}(t, a, p) d a d p & \\
& =\iiint_{p, p^{\prime}}\left(f\left(a, p^{\prime}\right)-f(a, p)\right) \Pi_{s-}^{\epsilon}\left(p, d p^{\prime}\right) P_{s-}^{\epsilon}(p) \rho^{\epsilon}(t, a, p) d a d p
\end{aligned}
$$

Rewritting the second member as follows, we have that

$$
\begin{gathered}
\iiint_{a, p, p^{\prime}}\left(f\left(a, p^{\prime}\right)-f(a, p)\right) \Pi_{s-}^{\epsilon}\left(p, d p^{\prime}\right) P_{s-}^{\epsilon}(p) \rho^{\epsilon}(t, a, p) d p d a \\
=\iiint_{p, p^{\prime}} C^{\epsilon}\left(f\left(a, p^{\prime}\right)-f(a, p)\right) \frac{\Psi^{\epsilon}\left(\left|p^{\prime}-p\right|\right) G_{s-}^{\epsilon}\left(p^{\prime}\right) d p^{\prime}}{\iiint \Psi^{\epsilon}(|q-p|) G_{s-}^{\epsilon}(q) d q} \frac{P_{s-}^{\epsilon}(p)}{C^{\epsilon}} \rho^{\epsilon}(t, a, p) d a d p= \\
=\iiint_{p}{ }^{t} \nabla f(a, p) \frac{C^{\epsilon} \iiint_{q} \Psi^{\epsilon}(|q-p|)(q-p) G_{s-}^{\epsilon}(q) d q}{\iiint \Psi^{\epsilon}(|q-p|) G_{s-}^{\epsilon}(q) d q} \frac{P_{s-}^{\epsilon}(p)}{C^{\epsilon}} \rho^{\epsilon}(t, a, p) d a d p+o(1) \\
=\iiint_{p}{ }^{t} \nabla f(a, p) \frac{C^{\epsilon} \iiint_{q} \Psi^{\epsilon}(|q-p|)(q-p)^{t}(q-p) d q}{\iiint \Psi^{\epsilon}(|q-p|) d q} \frac{P_{s-}^{\epsilon}(p)}{C^{\epsilon}} \rho^{\epsilon}(t, a, p) \frac{\nabla G_{s-}^{\epsilon}}{G_{s-}^{\epsilon}}(p) d a d p+o(1) \\
=\iiint_{p} \nabla f(a, p) \rho^{\epsilon}(t, a, p) C h(p) R^{t} \nabla(\log (1-R)) d p d a+o(1)
\end{gathered}
$$

By passing to the limit $\nabla f(a, p) \rho^{\epsilon}(t, a, p) C h(p)$ in $L^{2}$ and $\nabla(\log (1-R))$ in weak-* $L^{2}$ (see lemmaes 5.10-5.11 and [2]), we have that $\rho$ is a solution to

$$
\frac{\partial}{\partial t} \rho(t, a, p)+\frac{\partial}{\partial a} \rho(t, a, p)+\operatorname{div}(\rho(t, a, p) C h(p) R \nabla(\log (1-R)))=0, \quad \rho(t, 0, p)=\int B(a, p) \rho(t, a, p) d a,
$$

with $R=R\left(\int_{\mathbb{R}_{+}} \rho(t, a, p) d a \frac{V o l_{G}\left(3 r^{2}+6 r+3\right)}{3 r^{2}+6 r r_{O}(s)+3 r_{O}(s)^{2}}\right)$.

\subsection{Proof of theorem 2.2 Part III : Convergence of $Z_{t}^{M, \epsilon}$ as $M \rightarrow \infty$ and $\epsilon \rightarrow 0$}

Let $T>0$. Changing the time scale $t \mapsto t \epsilon$. We first notice that for $(M, \epsilon) \in U_{1}$, bounds given in the proof of theorem 2.2 Part I are independent of $\epsilon, M$. Indeed, the time scale appeared in lemma 5.5 that gives

$$
\sup _{M, \epsilon} \mathbb{E}\left(\sup _{t \in[0, T \epsilon]} \iiint \int r^{m} Z_{t}^{M, \epsilon}(d a, d p)\right)<\infty, \quad \forall T>0
$$


Then, changing the time scale, we find that all bounds given in Proof of theorem 2.2 Part I and in technica part 5.3.2 are in $O(T / \epsilon M)$ therefore uniform in $U_{1}$ (and so the tightness of $Z_{t}^{M, \epsilon}$ is assured in $U_{1}$ ). To obtain the convergence we need that $\epsilon M \rightarrow \infty$. Now, we construct a $\left(\epsilon_{k}, M_{k}\right)$ so that the limit is the one we are expecting ( i.e., $\rho$ ).

Since $C_{0}^{1}$ is a separable set, there exists $\left(\psi_{k}\right)_{k}$ dense subset of $C_{0}^{1}$. Let any sequence $\left(\epsilon_{k}\right)_{k}$ converging to 0 . Using theorem 2.2 , there exists $M^{1}$ s.t.

$$
\sup _{t \leq T}\left|\left\langle Z_{t}^{M^{1}, \epsilon_{1}}(d a, d p), \psi_{1}\right\rangle-\left\langle\rho_{\epsilon_{1}}(t, a, p) d a d p, \psi_{1}\right\rangle\right| \leq 1
$$

For the same reason, we can find $M^{2}$ s.t.

$$
\sup _{t \leq T}\left|\left\langle Z_{t}^{M^{2}, \epsilon_{2}}(d a, d p), \psi_{i}\right\rangle-\left\langle\rho_{\epsilon_{2}}(t, a, p) d a d p, \psi_{j}\right\rangle\right| \leq 1 / 4, j=1,2 .
$$

and so on, there exists $M^{n}$ s.t.

$$
\sup _{t \leq T}\left|\left\langle Z_{t}^{M^{n}, \epsilon_{n}}(d a, d p), \psi_{j}\right\rangle-\left\langle\rho_{\epsilon_{n}}(t, a, p) d a d p, \psi_{j}\right\rangle\right| \leq 1 / 2^{n}, j=1 . . n .
$$

Therefore, using the theorem 2.2 part I, we have that

$$
\lim _{n \rightarrow \infty} \sup _{t \leq T}\left|\left\langle Z_{t}^{M^{n}, \epsilon_{n}}(d a, d p), \psi\right\rangle-\langle\rho(t, a, p) d a d p, \psi\rangle\right|=0, \quad \forall \psi \in C_{0}^{1} .
$$

\section{Conclusion}

In this paper, we prove the convergence of a stochastic process which represent the evolution of a cell population as its population size goes to infinity and its cell size converges to zero. A way to understand the result is to imagine that we observe the evolution of the cell population at a certain distance (depending of the number of cells). If the distance is fixed (i.e. a fixed cell size), then when the number of cells goes to infinity, the whole space is filled by cells (we are too close) and we can only observe $Z_{t}^{M, \epsilon}=C$ st everywhere. If the distance is too far with respect to the size population, i.e. cells are too small, we observe a concentrated mass on the boundary of the oocyte (we are too far). To observe the cell population evolution we have to be, neither too close or too far, which explains the balance between $\epsilon$ (cell size, or observation distance to the follicule) and $M$ (cell population size). The proof is robust to the change of the birth rate $B$ and cell displacement rate (function $R$ ), as long as there are smooth and bounded. The next step is to study the dynamics of the final equation (15) and make the link with the partial differential equation of the follicle evolution given in $[19,6,7]$. 


\section{Annex}

\subsection{Regular approximation of $\chi_{\mathcal{L}_{i, j, k}^{\epsilon}}$}

We introduce a regular approximation of the characteristic function $\chi_{[0, \epsilon] \times[0, \pi / N] \times[0,2 \pi / N]}$ (see fig. 4). Let $\eta \in] 0,1[$ and

$$
\Psi^{\epsilon, \eta}:(r, \theta, \phi) \in \mathbb{R}^{3} \mapsto \Psi_{r}^{\epsilon, \eta}(r) \Psi_{\theta}^{\epsilon, \eta}(\theta) \Psi_{\phi}^{\epsilon, \eta}(\phi),
$$

a $C^{\infty}$ positive function such that $\Psi^{\epsilon, \eta} \leq 1$, such that

$$
\begin{gathered}
\left.\left(\Psi_{r}^{\epsilon}\right)\right|_{[\eta \epsilon, \epsilon(1-\eta)]}=1,\left.\quad \Psi_{r}^{\epsilon}\right|_{[-\eta \epsilon, \epsilon(1+\eta)]^{c}}=0, \\
\left.\left(\Psi_{\theta}^{\epsilon}\right)\right|_{\left[\frac{\eta}{N}, \frac{\pi-\eta}{N}\right]}=1,\left.\quad\left(\Psi_{\theta}^{\epsilon}\right)\right|_{\left[\frac{\pi+\eta}{N}, \pi-\frac{\eta}{N}\right]}=1, \quad \Psi_{\theta}^{\epsilon}(\theta+\pi)=\Psi_{\theta}^{\epsilon}(\theta), \quad \forall \theta \\
\left.\left(\Psi_{\phi}^{\epsilon}\right)\right|_{\left[-\pi+\frac{\eta}{N},-\pi+\frac{2 \pi-\eta}{N}\right]}=1,\left.\quad\left(\Psi_{\phi}^{\epsilon, \eta}\right)\right|_{\left[-\pi+\frac{2 \pi+\eta}{N}, \pi-\frac{\eta}{N}\right]}=1, \quad \Psi_{\phi}^{\epsilon}(\phi+2 \pi)=\Psi_{\phi}^{\epsilon}(\phi), \quad \forall \phi
\end{gathered}
$$

and satisfying the unity partition formulae

$$
\sum_{i, j, k} \Psi_{i, j, k}^{\epsilon}(r, \theta, \phi)=1, \quad \forall(r, \theta, \phi) \in[1+\eta \epsilon, \infty[\times[0, \pi] \times[-\pi, \pi] .
$$

with $\Psi_{i, j, k}^{\epsilon}$ a regular approximation of the characteristic function $\chi_{\mathcal{L}_{i, j, k}^{\epsilon}}$ :

$$
\Psi_{i, j, k}^{\epsilon}(r, \theta, \phi)=\Psi^{\epsilon}\left((r-1)-i \epsilon, \theta-\frac{j}{N}, \phi-\frac{k}{N}\right) .
$$

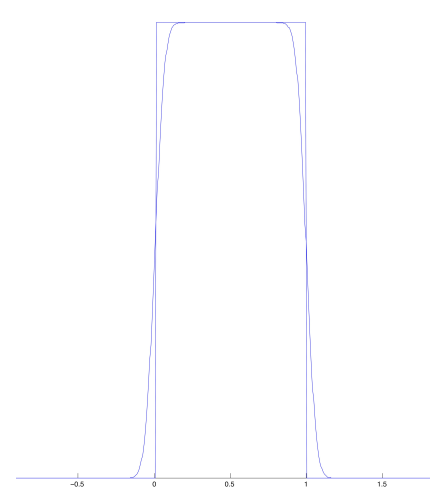

Figure 4: Regularization of characteristic function $\chi_{[0,1]}$. Using the convolution and Gaussian function it is easy to compute a regular approximation of $\chi_{[0,1]}$ which satisfies $(25)-(27)$.

\subsection{Proof of proposition 2.1 : Existence of the stochastic process $Z_{t}^{M, \epsilon}$}

This process can be construct step by step (see $[3,15,4,25,17]$ ), the only point is to prove global bounds

$$
0<E\left(\frac{N_{0}^{M, \epsilon}}{M}\right) \leq E\left(\sup _{s \leq t} N_{s}^{M, \epsilon}\right) \leq E\left(\frac{N_{0}^{M, \epsilon}}{M}\right) e^{t / M}<\infty,
$$




$$
0<r_{O}(0) \leq E\left(\sup _{s \leq t} r_{O}(s)\right) \leq r_{O}(0) e^{\frac{C}{\epsilon^{2}} E\left(\frac{N_{0}^{M, \epsilon}}{M}\right) e^{t / M} t}<\infty,
$$

where $N_{s}^{M, \epsilon}=\left\langle Z_{s}^{M, \epsilon}, 1\right\rangle$. Using (34), in the particular case $f=1$, we have that (using (9), we have $B_{n, s-}^{M, \epsilon} \leq$ $1<\infty)$

$$
N_{t}^{M, \epsilon}=\iint_{\mathbb{R}_{+} \times \text {Space }} 1 Z_{t}^{M, \epsilon}(d a, d p)=\frac{1}{M} N_{0}^{M, \epsilon}+\frac{1}{M} \int_{0}^{t} \int_{\varepsilon} 1_{n<N_{s-}^{M, \epsilon}}\left[11_{0 \leq \Theta<B_{n, s-}^{M, \epsilon}}\right] Q\left(d s,(d n), d \Theta, \Pi_{s-}^{M, \epsilon}\left(p, d p^{\prime}\right)\right) .
$$

Therefore, for $t \leq \tau_{N}^{M, \epsilon}$ (the stopping time $\tau_{N}^{M, \epsilon}$ is defined in $\left.(33)\right)$ and $N_{t}^{\bar{M}, \epsilon}=E\left(\sup _{s \leq \min \left(t, \tau_{N}^{M, \epsilon}\right)} N_{s}^{M, \epsilon}\right)$, we have that

$$
E\left(\frac{N_{0}^{M, \epsilon}}{M}\right) \leq N_{t}^{\bar{M}, \epsilon} \leq E\left(\frac{N_{0}^{M, \epsilon}}{M}\right)+\int_{0}^{t} \frac{1}{M} N_{s}^{\bar{M}, \epsilon} d s
$$

which implies (using Gronwall inequality on the right side) that

$$
E\left(\frac{N_{0}^{M, \epsilon}}{M}\right) \leq \bar{N}_{t} \leq E\left(\frac{N_{0}^{M, \epsilon}}{M}\right) e^{\frac{t}{M}}
$$

and so, we find that

$$
P\left(\inf _{M \geq N} \tau_{N}^{M, \epsilon} \geq t\right)=P\left(\tau_{N}^{M, \epsilon} \geq t\right)=P\left(\sup _{s \leq \min \left(t, \tau_{N}^{M, \epsilon}\right)} N_{s}^{M, \epsilon} \geq N\right) \leq E\left(\frac{N_{0}^{M, \epsilon}}{M}\right) e^{\frac{t}{M}} / N, P\left(\lim _{N \rightarrow \infty} \tau_{N}^{M, \epsilon}=\infty\right)=1 .
$$

Using Fatou lemma, we find that

$$
\left.\left.E\left(\lim _{N \rightarrow \infty} \sup _{s \leq \min \left(t, \tau_{N}^{M, \epsilon}\right)} N_{s}^{M, \epsilon}\right)\right)=E\left(\lim \inf _{N \rightarrow \infty} \sup _{s \leq \min \left(t, \tau_{N}^{M, \epsilon}\right)} N_{s}^{M, \epsilon}\right)\right) \leq \lim \inf _{N \rightarrow \infty} N_{t}^{\bar{M}, \epsilon} \leq E\left(\frac{N_{0}^{M, \epsilon}}{M}\right) e^{\frac{t}{M}},
$$

and finally $E\left(\sup _{s \leq t} N_{s}^{M, \epsilon}\right) \leq E\left(\frac{N_{0}^{M, \epsilon}}{M}\right) e^{\frac{t}{M}}$. Similarly, we have (30). Let $\left(T_{k}\right)_{k}$ the sequences of successive jumps of the $Z_{s}^{M, \epsilon}$ process, then, $\lim _{k} T_{k}=\infty$, a.e. Indeed, let $U_{M} \subset \Omega$, such that $\lim _{k} T_{k}(\omega)<M$ for $\omega \in U_{M}$, then necessarily $\lim _{k \rightarrow \infty} N_{T_{k}}^{M, \epsilon}(\omega)<\infty$ (otherwise $\tau_{N}^{M, \epsilon} \leq M / 2$ for $N$ large enough, which is with null probability) and so, for all $\omega \in U_{M}$, we can construct the time sequence $\left(T_{k}(\omega)\right)_{k}$ as a subsequence of a Poisson point process of intensity $\left(1+C / \epsilon^{2}\right) N_{\infty}$ (using (9) and (10) we have that $1+C / \epsilon^{2}<\infty$ ) where $N_{\infty}=\lim _{k \rightarrow \infty} N_{T_{k}}(\omega)$ which is a.e. unbounded. Moreover, we have for all $\epsilon>0, F$ and $f$ belongs to $W^{1, \infty}$, the infinitesimal generator of the Markovian process $\left(Z_{t}^{M, \epsilon}\right)_{t \geq 0}$ issued from $Z_{0}^{M, \epsilon}$ is given by

$$
\begin{aligned}
\mathcal{L} F_{f}\left(Z_{0}^{M, \epsilon}\right)=\frac{\partial}{\partial t} & \left.\mathbb{E}\left(F\left(\left\langle f, Z_{t}^{M, \epsilon}\right\rangle\right)\right)\right|_{t=0}=F^{\prime}\left(\left\langle f, Z_{0}^{M, \epsilon}\right\rangle\right) \iint_{\mathbb{R}_{+} \times S p a c e} \frac{\partial}{\partial a} f(a, p) Z_{0}^{M, \epsilon}(d a, d p) \\
+ & \left(\int \int _ { \mathbb { R } _ { + } \times S p a c e } \left[\left(F\left(\left\langle f, Z_{0}^{M, \epsilon}\right\rangle+\frac{2}{M} f(0, p)-\frac{1}{M} f(a, p)\right)-F\left(\left\langle f, Z_{0}^{M, \epsilon}\right\rangle\right) B(0, a)\right.\right.\right. \\
& \left.+\left(F\left(\left\langle f, Z_{0}^{M, \epsilon}\right\rangle+\frac{1}{M} f\left(a, p^{\prime}\right)-\frac{1}{M} f(a, p)\right)-F\left(\left\langle f, Z_{0}^{M, \epsilon}\right\rangle\right)\right) P_{0}^{\epsilon} \Pi_{0}^{\epsilon}\left(p, d p^{\prime}\right)\right] Z_{0}^{M, \epsilon}(d a, d p) .
\end{aligned}
$$

The infinitesimal generator of the Markovian process $\left(r_{O}^{M, \epsilon}(t)\right)_{t \geq 0}$ issued from $r_{0}$ is given by

$$
\mathcal{L} F\left(r_{O}^{M, \epsilon}(0)\right)=\left.\frac{\partial}{\partial t} \mathbb{E}\left(F\left(r_{O}^{M, \epsilon}(t)\right)\right)\right|_{t=0}=F^{\prime}\left(r_{0}\right) \sum_{i, j, k} \kappa((i-1) \epsilon) r_{0}^{\alpha}\left\langle\Psi_{i, j, k}^{\epsilon}, Z_{0}^{M, \epsilon}\right\rangle .
$$


Indeed, let $t \leq \tau_{N}^{M, \epsilon}$ with $\tau_{N}^{M, \epsilon}=\inf \left\{s \geq 0, \quad N_{s}^{M, \epsilon} \geq N\right\}$, then using lemma 5.2, we have that

$$
\begin{gathered}
\mathbb{E}\left(\left(\int_{0}^{\min \left(t, \tau_{N}^{M, \epsilon}\right)} F^{\prime}\left(\left\langle f, Z_{u}^{M, \epsilon}\right\rangle\right) \iint_{\mathbb{R}_{+} \times \text {Space }} \frac{\partial}{\partial a} f(u, a, p) Z_{u}^{M, \epsilon}(d a, d p) d u\right)=\right. \\
\mathbb{E}\left(F\left(\iint_{\mathbb{R}_{+} \times \text {Space }} f(a, p) Z_{t}^{M, \epsilon}(d a, d p)\right)\right)-\mathbb{E}\left(F\left(\iint_{\mathbb{R}_{+} \times \text {Space }} f(a, p) Z_{0}(d a, d p)\right)\right) \\
-\left[\mathbb { E } \left(\int _ { 0 } ^ { \operatorname { m i n } ( t , \tau _ { N } ^ { M , \epsilon } ) } \int _ { \varepsilon } 1 _ { n < N _ { s - } ^ { M , \epsilon } } \left[\left(F\left(\left\langle f, Z_{s}\right\rangle+\frac{2}{M} f\left(0, X_{n}^{M, \epsilon}(s-)\right)-\frac{1}{M} f\left(A_{n}^{M, \epsilon}(s-), X_{n}^{M, \epsilon}(s-)\right)\right)-F\left(\left\langle f_{s}, Z_{s}^{M, \epsilon}\right\rangle\right)\right.\right.\right.\right. \\
1_{0 \leq \Theta<B_{n, s-}^{M, \epsilon}}+\left(F\left(\left\langle f, Z_{s}\right\rangle+\frac{1}{M} f\left(A_{n}^{M, \epsilon}(s-), p^{\prime}\right)-\frac{1}{M} f\left(A_{n}^{M, \epsilon}(s-), X_{n}^{M, \epsilon}(s-)\right)\right)-F\left(\left\langle f, Z_{s}\right\rangle\right)\right) \\
\left.\left.\left.\left.1_{0 \leq \Theta-B_{n, s-}^{M, \epsilon}<P_{s-}^{\epsilon}\left(X_{n}(s-)\right)}\right] Q\left(d s,(d n), d \Theta, \Pi_{s-}^{M, \epsilon}\left(p, d p^{\prime}\right)\right)\right)\right)\right]
\end{gathered}
$$

which can be rewritten (for simplicity) as

$$
\mathbb{E}\left(F\left(\iint_{\mathbb{R}_{+} \times \text {Space }} f(a, p) Z_{\min \left(t, \tau_{N}^{M, \epsilon}\right)}(d a, d p)\right)\right)=\mathbb{E}\left(F\left(\iint_{\mathbb{R}_{+} \times \text {Space }} f(a, p) Z_{0}(d a, d p)\right)\right)+\mathbb{E}\left(\Psi\left(\min \left(t, \tau_{N}^{M, \epsilon}\right), Z\right)\right),
$$

with

$$
\begin{aligned}
\Psi(t, Z)= & \int_{0}^{t} F^{\prime}\left(\left\langle f, Z_{u}^{M, \epsilon}\right\rangle\right) \iint_{\mathbb{R}_{+} \times \text {Space }} \frac{\partial}{\partial a} f(a, p) Z_{u}^{M, \epsilon}(d a, d p) d u \\
& +\left(\int _ { 0 } ^ { t } \int \int _ { \mathbb { R } _ { + } \times \text { Space } } 1 _ { n < N _ { s - } ^ { M , \epsilon } } \left[\left(F\left(\left\langle f, Z_{s}\right\rangle+\frac{2}{M} f(s, 0, p)-\frac{1}{M} f(s, a, p)\right)-F\left(\left\langle f, Z_{s}\right\rangle\right) B(p, a)\right.\right.\right. \\
& \left.\quad+\left(F\left(\left\langle f, Z_{s}\right\rangle+\frac{1}{M} f\left(s, a, p^{\prime}\right)-\frac{1}{M} f(s, a, p)\right)-F\left(\left\langle f, Z_{s}\right\rangle\right)\right) P_{s-}^{\epsilon}(p) \Pi_{s-}^{\epsilon}\left(p, d p^{\prime}\right)\right] Z_{s}^{M, \epsilon}(d a, d p)
\end{aligned}
$$

with $B(p, a)=1-e^{-a / \lambda\left(\|p\|_{2}\right)}$. We have the following bound

$$
\left|\Psi\left(\min \left(t, \tau_{N}^{M, \epsilon}\right), Z\right)\right| \leq T N\|F\|_{W^{1, \infty}}\left(1+\|f\|_{W^{1, \infty}}\left(1+\sup _{p} \int\left|p^{\prime}-p\right| P^{\epsilon}(p) \Pi_{s-}\left(p, d p^{\prime}\right)\right)\right) .
$$

and since $Z_{t}^{M, \epsilon}$ is càd and $\tau_{N}^{M, \epsilon}>0$, we have

$$
\begin{aligned}
& \left.\frac{\partial}{\partial t} \Psi\left(\min \left(t, \tau_{N}^{M, \epsilon}\right), Z\right)\right|_{t=0}=F^{\prime}\left(\left\langle f_{0}, Z_{0}^{M, \epsilon}\right\rangle\right) \iint_{\mathbb{R}_{+} \times \text {Space }} \frac{\partial}{\partial a} f(a, p) Z_{0}^{M, \epsilon}(d a, d p) \\
& +\left(\int \int _ { \mathbb { R } _ { + } \times \text { Space } } \left[\left(F\left(\left\langle f, Z_{0}\right\rangle+\frac{2}{M} f(0,0, p)-\frac{1}{M} f(0, a, p)\right)-F\left(\left\langle f, Z_{0}^{M, \epsilon}\right\rangle\right) B(0, a)\right.\right.\right. \\
& \left.\quad+\left(F\left(\left\langle f, Z_{0}\right\rangle+\frac{1}{M} f\left(0, a, p^{\prime}\right)-\frac{1}{M} f(0, a, p)\right)-F\left(\left\langle f, Z_{0}^{M, \epsilon}\right\rangle\right)\right) P_{0}^{\epsilon}(p) \Pi_{0}^{\epsilon}\left(p, d p^{\prime}\right)\right] Z_{0}^{M, \epsilon}(d a, d p)
\end{aligned}
$$

which is dominated by $T \frac{N_{0}^{M, \epsilon}}{M}\|F\|_{W^{1, \infty}}\left(1+\|f\|_{W^{1, \infty}}(1+C / \epsilon)\right)<\infty$. Therefore by derivation under domination, we find (31).

\subsection{Technical lemmaes : Stochastic}

In this part, we give the doob decomposition of the stochastic process $Z_{s}^{M, \epsilon}$ (issued from Ito calculus). Then, we give uniform bounds which are used to prove the tightness of $Z_{s}^{M, \epsilon}$. 


\subsubsection{Ito calculus and first lemmas}

For convenience, we introduced stopping time $\left(\tau_{N}^{M, \epsilon}\right)$

$$
\tau_{N}^{M, \epsilon}=\inf \left\{s \geq 0, \quad\left\langle 1, Z_{s}^{M, \epsilon} \wedge r_{O}^{M, \epsilon}(s)\right\rangle \geq N\right\} .
$$

We first give computational lemmas on the population evolution.

Lemma 5.1 For all $f \in C^{0}($ Space $\times$ Age $), t>0$ and $r_{O}, Z_{t}^{M, \epsilon}$ solution to (7)-(8) we have

$$
\begin{aligned}
& \iint_{\mathbb{R}_{+} \times \text {Space }} f(t, a, p) Z_{t}^{M, \epsilon}(d a, d p)=\frac{1}{M} \sum_{k=1}^{N_{0}^{M, \epsilon}} f\left(t, A_{n}^{M, \epsilon}(0)+t, X_{n}^{M, \epsilon}(0)\right) \\
&+\frac{1}{M} \int_{0}^{t} \int_{\varepsilon} 1_{n<N_{s-}^{M, \epsilon}}\left[\left(2 f\left(t, t-s, X_{n}^{M, \epsilon}(s-)\right)-f\left(t, A_{n}^{M, \epsilon}(s-)+t-s, X_{n}^{M, \epsilon}(s-)\right)\right) 1_{0 \leq \Theta<B_{n, s-}^{M, \epsilon}}\right. \\
&\left.+\left(f\left(t, A_{n}^{M, \epsilon}(s-)+t-s, p^{\prime}\right)-f\left(t, A_{n}^{M, \epsilon}(s-)+t-s, X_{n}^{M, \epsilon}(s-)\right)\right) 1_{0 \leq \Theta-B_{n, s-}^{M, \epsilon}<P_{s-}^{\epsilon}\left(X_{n}(s-)\right)}\right] \\
& Q\left(d s,(d n), d \Theta, \Pi_{s-}^{M, \epsilon}\left(p, d p^{\prime}\right)\right)
\end{aligned}
$$

Proof Direct computation (see [4] for more details).

Lemma 5.2 Let $F \in C^{1}(\mathbb{R}, \mathbb{R})$ and $f \in \mathcal{B}\left(\mathbb{R}_{+} \times \mathbb{R}_{+} \times\right.$Space, $\left.\mathbb{R}\right)$ s.t. $\forall p \in$ Space, $\quad(s, a) \in \mathbb{R}_{+}^{2} \mapsto f(s, a, p) \in$ $C_{b}^{1,1}\left(\mathbb{R}_{+} \times\right.$Space, $\left.\mathbb{R}\right)$ with uniform (in space) bounds of the partial derivation of $f$ then, for all $t>0$,

$$
\begin{gathered}
\int_{0}^{t} F^{\prime}\left(\left\langle f_{u}, Z_{u}^{M, \epsilon}\right\rangle\right) \iint_{\mathbb{R}_{+} \times S p a c e}\left(\frac{\partial}{\partial u}+\frac{\partial}{\partial a}\right) f(u, a, p) Z_{u}^{M, \epsilon}(d a, d p) d u=F\left(\left\langle f_{t}, Z_{t}^{M, \epsilon}\right\rangle\right)-F\left(\left\langle f_{0}, Z_{0}^{M, \epsilon}\right\rangle\right) \\
-\left[\int _ { 0 } ^ { t } \int _ { \varepsilon } 1 _ { n < N _ { s - } ^ { M , \epsilon } } \left[\left(F\left(\left\langle f_{s}, Z_{s}\right\rangle+\frac{2}{M} f\left(s, 0, X_{n}^{M, \epsilon}(s-)\right)-\frac{1}{M} f\left(s, A_{n}^{M, \epsilon}(s-), X_{n}^{M, \epsilon}(s-)\right)\right)-F\left(\left\langle f_{s}, Z_{s}^{M, \epsilon}\right\rangle\right) 1_{0 \leq \Theta<B_{n, s-}^{M, \epsilon}}\right.\right.\right. \\
+\left(F\left(\left\langle f_{s}, Z_{s}\right\rangle+\frac{1}{M} f\left(s, A_{n}^{M, \epsilon}(s-), p^{\prime}\right)-\frac{1}{M} f\left(s, A_{n}^{M, \epsilon}(s-), X_{n}^{M, \epsilon}(s-)\right)\right)-F\left(\left\langle f_{s}, Z_{s}\right\rangle\right)\right) \\
\left.\left.1_{0 \leq \Theta-B_{n, s-}^{M, \epsilon}<P_{s-}^{\epsilon}\left(X_{n}(s-)\right)}\right] Q\left(d s,(d n), d \Theta, \Pi_{s-}^{M, \epsilon}\left(p, d p^{\prime}\right)\right)\right]
\end{gathered}
$$

with $\left\langle f_{s}, Z_{s}^{M, \epsilon}\right\rangle=\iint_{\mathbb{R}_{+} \times \text {Space }} f(s, a, p) Z_{s}^{M, \epsilon}(d a, d p)$.

Proof Using (34) of lemma 5.1, in the particular case $\left(\frac{\partial}{\partial u}+\frac{\partial}{\partial a}\right) f(u, a, p)$, and integrating in time, we have that $Z_{t}^{M, \epsilon}$ satisfies (for all $f$ given in assumptions)

$$
\begin{gathered}
\int_{0}^{t} \iint_{\mathbb{R}_{+} \times \text {Space }}\left(\frac{\partial}{\partial u}+\frac{\partial}{\partial a}\right) f(u, a, p) Z_{u}^{M, \epsilon}(d a, d p) d u=\frac{1}{M} \int_{0}^{t} \sum_{k=1}^{N_{0}^{M, \epsilon}}\left(\frac{\partial}{\partial u}+\frac{\partial}{\partial a}\right) f\left(u, A_{n}^{M, \epsilon}(0)+u, X_{n}^{M, \epsilon}(0)\right) d u \\
+\frac{1}{M} \int_{0}^{t} \int_{0}^{u} \int_{\varepsilon} 1_{n<N_{s-}^{M, \epsilon}}\left[\left(2\left(\frac{\partial}{\partial u}+\frac{\partial}{\partial a}\right) f\left(u, u-s, X_{n}^{M, \epsilon}(s-)\right)\right.\right. \\
\left.\quad-\left(\frac{\partial}{\partial u}+\frac{\partial}{\partial a}\right) f\left(u, A_{n}^{M, \epsilon}(s-)+u-s, X_{n}^{M, \epsilon}(s-)\right)\right) 1_{0 \leq \Theta<B_{n, s-}^{M, \epsilon}} \\
+\left(\left(\frac{\partial}{\partial u}+\frac{\partial}{\partial a}\right) f\left(u, A_{n}^{M, \epsilon}(s-)+u-s, p^{\prime}\right)-f\left(u, A_{n}^{M, \epsilon}(s-)+u-s, X_{n}^{M, \epsilon}(s-)\right)\right) \\
\left.1_{0 \leq \Theta-B_{n, s-}^{M, \epsilon}<P_{s-}^{\epsilon}\left(X_{n}(s-)\right)}\right] Q\left(d s,(d n), d \Theta, \Pi_{s-}^{M, \epsilon}\left(p, d p^{\prime}\right)\right) d u .
\end{gathered}
$$


Now, using Fubinni theorem $\int_{u=0}^{t} \int_{s=0}^{u}=\int_{s=0}^{t} \int_{u=s}^{t}$ and $\left(\left(\frac{\partial}{\partial u}+\frac{\partial}{\partial a}\right) f\right)(u, .+u,)=.\frac{d}{d u}(f(u, .+u,)$.$) , we have that$

$$
\begin{gathered}
\int_{0}^{t} \iint_{\mathbb{R}_{+} \times \text {Space }}\left(\frac{\partial}{\partial u}+\frac{\partial}{\partial a}\right) f(u, a, p) Z_{u}^{M, \epsilon}(d a, d p) d u=\frac{1}{M} \sum_{k=1}^{N_{0}^{M, \epsilon}}\left[f\left(t, A_{n}^{M, \epsilon}(0)+t, X_{n}^{M, \epsilon}(0)\right)-f\left(0, A_{n}^{M, \epsilon}(0), X_{n}^{M, \epsilon}(0)\right)\right] \\
\quad+\frac{1}{M} \int_{0}^{t} \int_{\varepsilon} 1_{n<N_{s-}^{M, \epsilon}}\left[\left(2\left(f\left(t, t-s, X_{n}^{M, \epsilon}(s-)\right)-f\left(s, 0, X_{n}^{M, \epsilon}(s-)\right)\right)\right.\right. \\
\left.\left.-\left(f\left(t, A_{n}^{M, \epsilon}(s-)+t-s, X_{n}^{M, \epsilon}(s-)\right)\right)-f\left(s, A_{n}^{M, \epsilon}(s-), X_{n}^{M, \epsilon}(s-)\right)\right)\right) 1_{0 \leq \Theta<B_{n, s-}^{M, \epsilon}} \\
\left.+\left(f\left(t, A_{n}^{M, \epsilon}(s-)+t-s, p^{\prime}\right)-f\left(s, A_{n}^{M, \epsilon}(s-), J\right)\right)-\left(f\left(t, A_{n}^{M, \epsilon}(s-)+t-s, X_{n}^{M, \epsilon}(s-)\right)-f\left(s, A_{n}^{M, \epsilon}(s-), X_{n}^{M, \epsilon}(s-)\right)\right)\right) \\
\left.1_{0 \leq \Theta-B_{n, s-}^{M, \epsilon}<P_{s-}^{\epsilon}\left(X_{n}(s-)\right)}\right] d u Q\left(d s,(d n), d \Theta, \Pi_{s-}^{M, \epsilon}\left(p, d p^{\prime}\right)\right) .
\end{gathered}
$$

Using formula (34), we find finally that

$$
\begin{aligned}
& \int_{0}^{t} \iint_{\mathbb{R}_{+} \times \text {Space }}\left(\frac{\partial}{\partial u}+\frac{\partial}{\partial a}\right) f(u, a, p) Z_{u}^{M, \epsilon}(d a, d p) d u=\left\langle f_{t}, Z_{t}^{M, \epsilon}\right\rangle-\left\langle f_{0}, Z_{0}^{M, \epsilon}\right\rangle \\
&- \frac{1}{M}\left[\int _ { 0 } ^ { t } \int _ { \varepsilon } 1 _ { n < N _ { s - } ^ { M , \epsilon } } \left(\left(2 f\left(s, 0, X_{n}^{M, \epsilon}(s-)\right)-f\left(s, A_{n}^{M, \epsilon}(s-), X_{n}^{M, \epsilon}(s-)\right)\right) 1_{0 \leq \Theta<B_{n, \epsilon-}^{M, \epsilon}}\right.\right. \\
&\left.+\left(f\left(s, A_{n}^{M, \epsilon}(s-), p^{\prime}\right)-f\left(s, A_{n}^{M, \epsilon}(s-), X_{n}^{M, \epsilon}(s-)\right)\right) 1_{0 \leq \Theta-B_{n, s-}^{M, \epsilon}<P_{s-}^{\epsilon}\left(X_{n}(s-)\right)}\right) \\
&\left.Q\left(d s,(d n), d \Theta, \Pi_{s-}^{M, \epsilon}\left(p, d p^{\prime}\right)\right)\right] .
\end{aligned}
$$

Now, using the Itô formula with jump processes, we find that $(35)$ is satisfied (see $[4,25,17,15,3]$ for more details).

\subsubsection{Doob decomposition of $Z_{t}^{M, \epsilon}$}

Let $m \geq 1$ and assume that

$$
\sup _{M, \epsilon} \mathbb{E}\left(\left\langle 1+a^{2 m}+r^{2 m}, Z_{0}^{M, \epsilon}(d a, d p)\right\rangle+\left\langle 1+a^{m}+r^{m}, Z_{0}^{M, \epsilon}(d a, d p)\right\rangle^{2}\right)<\infty
$$

then we have that for all $T>0$ (see lemmaes 5.3-5.7),

$$
\sup _{M, \epsilon} \mathbb{E}\left(\sup _{t \in[0, T \epsilon]}\left\langle 1+a^{2 m}+r^{2 m}, Z_{t}^{M, \epsilon}(d a, d p)\right\rangle+\sup _{t \in[0, T \epsilon]}\left\langle 1+a^{m}+r^{m}, Z_{t}^{M, \epsilon}(d a, d p)\right\rangle^{2}\right)<\infty .
$$

Moreover, for all $f$,

$$
\left\langle f_{t}, Z_{t}^{M, \epsilon}\right\rangle=\mathcal{M}_{t}^{M, \epsilon}(f)+\mathcal{V}_{t}^{M, \epsilon}(f)
$$

where

$$
\begin{aligned}
\mathcal{M}_{t}^{M, \epsilon}(f) & =\left\langle f_{t}, Z_{t}^{M, \epsilon}\right\rangle-\left\langle f_{0}, Z_{0}^{M, \epsilon}\right\rangle-\int_{0}^{t}\left\langle\left(\frac{\partial}{\partial u}+\frac{\partial}{\partial a}\right) f(u, a, p), Z_{u}^{M, \epsilon}(d a, d p)\right\rangle d u \\
& -\int_{0}^{t}\left\langle(2 f(s, 0, p)-f(s, a, p)) B(a, p)+\int_{\text {Space }}\left(f\left(s, p, p^{\prime}\right)-f(s, a, p)\right) P_{s-}^{\epsilon}(p) \Pi_{s-}^{\epsilon}\left(p, d p^{\prime}\right), Z_{s}^{M, \epsilon}\right\rangle d s,
\end{aligned}
$$

is an $L^{2}$ martingale càdlàg nul at $t=0$ of quadratic previsible increasing process

$$
\begin{aligned}
& \left\langle\mathcal{M}_{t}^{M, \epsilon}(f)\right\rangle= \\
& \quad \frac{1}{M} \int_{0}^{t}\left\langle(2 f(s, 0, p)-f(s, a, p))^{2} B(a, p)+\int_{\text {Space }}\left(f\left(s, a, p^{\prime}\right)-f(s, a, p)\right)^{2} P_{s-}^{\epsilon} \Pi_{s-}^{\epsilon}\left(p, d p^{\prime}\right), Z_{s}^{M, \epsilon}\right\rangle d s,
\end{aligned}
$$


and

$$
\begin{aligned}
\mathcal{V}_{t}^{M, \epsilon}(f) & =\left\langle f_{0}, Z_{0}^{M, \epsilon}\right\rangle+\int_{0}^{t}\left\langle\left(\frac{\partial}{\partial u}+\frac{\partial}{\partial a}\right) f(u, a, p), Z_{u}^{M, \epsilon}(d a, d p)\right\rangle d u \\
+ & \int_{0}^{t}\left\langle(2 f(s, 0, p)-f(s, a, p)) B(a, p)+\int_{\text {Space }}\left(f\left(s, p, p^{\prime}\right)-f(s, a, p)\right) P_{s-}^{\epsilon}(p) \Pi_{s-}^{\epsilon}\left(p, d p^{\prime}\right), Z_{s}^{M, \epsilon}\right\rangle d s,
\end{aligned}
$$

which satisfy in average (in $d \mathcal{P}(\omega))$ an uniform $W_{l o c}^{1, \infty}\left(\mathbb{R}_{+}\right)$bound. More precisely, for all stopping time $T, S$

$$
\begin{gathered}
\mathbb{E}\left(\sup _{t \leq T}\left|\left\langle\mathcal{M}_{t}^{M, \epsilon}(f)\right\rangle\right|\right) \leq\left(9\|f\|_{L^{\infty}}^{2}+\|f\|_{W^{1, \infty}}^{2}(1+C)\right) \mathbb{E}\left(\frac{|T|}{M} \sup _{s \leq T}\left\langle 1, Z_{s}^{M, \epsilon}\right\rangle\right), \\
\mathbb{E}\left(\left|\left\langle\mathcal{M}_{T}^{M, \epsilon}(f)\right\rangle-\left\langle\mathcal{M}_{S}^{M, \epsilon}(f)\right\rangle\right|\right) \leq\left(9\|f\|_{L^{\infty}}^{2}+\|f\|_{W^{1, \infty}}^{2}(1+C)\right) \mathbb{E}\left(\frac{|T-S|}{M} \sup _{s \leq \max (T, S)}\left\langle 1, Z_{s}^{M, \epsilon}\right\rangle\right), \\
\mathbb{E}\left(\sup _{t \leq T}\left|\left\langle\mathcal{V}_{t}^{M, \epsilon}(f)\right\rangle\right|\right) \leq \mathbb{E}\left(\mid\left\langle f_{0}, Z_{0}^{M, \epsilon}\right|\right)+\left(3\|f\|_{L^{\infty}}+\|f\|_{W^{1, \infty}}(1+C)\right)\left(1+\mathbb{E}\left(|T| \sup _{s \leq T}\left\langle 1, Z_{s}^{M, \epsilon}\right\rangle\right)\right),
\end{gathered}
$$

and

$$
\mathbb{E}\left(\left|\left\langle\mathcal{V}_{T}^{M, \epsilon}(f)\right\rangle-\left\langle\mathcal{V}_{S}^{M, \epsilon}(f)\right\rangle\right|\right) \leq\left(3\|f\|_{L^{\infty}}+\|f\|_{W^{1, \infty}}(1+C)\right) \mathbb{E}\left(\frac{|T-S|}{M}\left(1+\sup _{s \leq \max (T, S)}\left\langle 1, Z_{s}^{M, \epsilon}\right\rangle\right)\right) .
$$

Proof To prove bound (38), we use technical lemmas 5.3-5.7 (proves are similar to [26, 17]). Now, for all $f \in C^{0}($ Space $\times$ Age $), t>0$ and $r_{O}, Z_{t}^{M, \epsilon}$ solution to (7)-(8) we have (see lemma 5.2)

$$
\begin{aligned}
& 0=\left\langle f_{t}, Z_{t}^{M, \epsilon}\right\rangle-\left\langle f_{0}, Z_{0}^{M, \epsilon}\right\rangle-\int_{0}^{t}\left\langle\left(\frac{\partial}{\partial u}+\frac{\partial}{\partial a}\right) f(u, a, p), Z_{u}^{M, \epsilon}(d a, d p)\right\rangle d u \\
& -\frac{1}{M}\left[\int _ { 0 } ^ { t } \int _ { \varepsilon } 1 _ { n < N _ { s - } ^ { M , \epsilon } } \left[\left(2 f\left(s, 0, X_{n}^{M, \epsilon}(s-)\right)-f\left(s, A_{n}^{M, \epsilon}(s-), X_{n}^{M, \epsilon}(s-)\right)\right) 1_{0 \leq \Theta<B\left(A_{n}^{M}(s-), X_{n}^{M}(s-)\right)}\right.\right. \\
& +\left(f\left(s, A_{n}^{M, \epsilon}(s-), p^{\prime}\right)-f\left(s, A_{n}^{M, \epsilon}(s-), X_{n}^{M, \epsilon}(s-)\right)\right) \\
& \left.\left.1_{B\left(A_{n}^{M}(s-), X_{n}^{M}(s-)\right) \leq \Theta<B\left(A_{n}^{M}(s-), X_{n}^{M}(s-)\right)+P_{s-}^{\epsilon}\left(X_{n}^{M}(s-)\right)}\right] Q\left(d s,(d n), d \Theta, \Pi_{s-}^{\epsilon}\left(p, d p^{\prime}\right)\right)\right]
\end{aligned}
$$

Using (34), we have that

$$
\begin{aligned}
& \mathcal{M}_{t}^{M, \epsilon}(f)=-\frac{1}{M} \int_{0}^{t} \int_{\varepsilon} 1_{n<N_{s-}^{M, \epsilon}}\left[\left(2 f\left(s, 0, X_{n}^{M, \epsilon}(s-)\right)-f\left(s, A_{n}^{M, \epsilon}(s-), X_{n}^{M, \epsilon}(s-)\right)\right) 1_{0 \leq \Theta<B\left(A_{n}^{M}(s-), X_{n}^{M}(s-)\right)}\right. \\
& +\left(f\left(s, A_{n}^{M, \epsilon}(s-), p^{\prime}\right)-f\left(s, A_{n}^{M, \epsilon}(s-), X_{n}^{M, \epsilon}(s-)\right)\right) \\
& \left.1_{B\left(A_{n}^{M}(s-), X_{n}^{M}(s-)\right) \leq \Theta<B\left(A_{n}^{M}(s-), X_{n}^{M}(s-)\right)+P_{s-}^{\epsilon}\left(X_{n}^{M}(s-)\right)}\right] \tilde{Q}\left(d s,(d n), d \Theta, \Pi_{s-}^{\epsilon}\left(p, d p^{\prime}\right)\right)
\end{aligned}
$$

with $\tilde{Q}=Q-d s \otimes \operatorname{Compt}(d n) \otimes d \Theta \otimes \Pi_{s-}^{M, \epsilon}\left(p, d p^{\prime}\right)$ be the compensated Poisson process of $Q$ introduced in the introduction. Therefore $\mathcal{M}_{t}^{M, \epsilon}(f)$ is a local martingale associated to the stopping times sequence $\left(\tau_{N}^{M, \epsilon}\right)_{N}$ introduced in (33). Using Ito formula, we have that

$$
\begin{aligned}
& \mathcal{M}_{t}^{M, \epsilon}(f)^{2}=-\int_{0}^{t} \int_{\varepsilon} 1_{n<N_{s-}^{M, \epsilon}} \\
& {\left[\left(\left(\left\langle f_{s}, Z_{s}^{M, \epsilon}\right\rangle+\frac{2}{M} f\left(s, 0, X_{n}^{M, \epsilon}(s-)\right)-\frac{1}{M} f\left(s, A_{n}^{M, \epsilon}(s-), X_{n}^{M, \epsilon}(s-)\right)\right)^{2}-\left\langle f_{s}, Z_{s}^{M, \epsilon}\right\rangle^{2}\right) 1_{0 \leq \Theta<B\left(A_{n}^{M}(s-), X_{n}^{M}(s-)\right)}\right.} \\
& +\left(\left(\left\langle f_{s}, Z_{s}^{M, \epsilon}\right\rangle+\frac{1}{M} f\left(s, A_{n}^{M, \epsilon}(s-), p^{\prime}\right)-\frac{1}{M} f\left(s, A_{n}^{M, \epsilon}(s-), X_{n}^{M, \epsilon}(s-)\right)\right)^{2}-\left\langle f_{s}, Z_{s}^{M, \epsilon}\right\rangle^{2}\right) \\
& \left.1_{B\left(A_{n}^{M}(s-), X_{n}^{M}(s-)\right) \leq \Theta<B\left(A_{n}^{M}(s-), X_{n}^{M}(s-)\right)+P_{s-}^{\epsilon}\left(X_{n}^{M}(s-)\right)}\right] \tilde{Q}\left(d s,(d n), d \Theta, \Pi_{s-}^{\epsilon}\left(p, d p^{\prime}\right)\right)
\end{aligned}
$$


Using lemma 5.2, we find that

$$
\begin{aligned}
& \left\langle f_{t}, Z_{t}^{M, \epsilon}\right\rangle^{2}-\left\langle f_{0}, Z_{0}^{M, \epsilon}\right\rangle^{2}-2 \int_{0}^{t}\left\langle f_{u}, Z_{u}^{M, \epsilon}\right\rangle\left\langle\left(\frac{\partial}{\partial u}+\frac{\partial}{\partial a}\right) f(u, a, p), Z_{u}^{M, \epsilon}(d a, d p)\right\rangle d u \\
& -2 \int_{0}^{t}\left\langle f_{s}, Z_{s}^{M, \epsilon}\right\rangle\left\langle(2 f(s, 0, p)-f(s, a, p)) B(a, p)+\int_{\text {Space }}\left(f\left(s, a, p^{\prime}\right)-f(s, a, p)\right) P_{s-}^{\epsilon}(p) \Pi_{s-}^{\epsilon}\left(p, d p^{\prime}\right), Z_{s}^{M, \epsilon}\right\rangle d s \\
& -\frac{1}{M} \int_{0}^{t}\left\langle(2 f(s, 0, p)-f(s, a, p))^{2} B(a, p)+\int_{\text {Space }}\left(f\left(s, a, p^{\prime}\right)-f(s, a, p)\right)^{2} P_{s-}^{\epsilon}(p) \Pi_{s-}^{\epsilon}\left(p, d p^{\prime}\right), Z_{s}^{M, \epsilon}\right\rangle d s=-\mathcal{M}_{t}^{M, \epsilon}(f)^{2},
\end{aligned}
$$

which means that

$$
\begin{aligned}
& \mathcal{M}_{t}^{M, \epsilon}(f)^{2}=\text { Local }_{\text {Martingale }} \\
& \quad+\frac{1}{M} \int_{0}^{t}\left\langle(2 f(s, 0, p)-f(s, a, p))^{2} B(a, p)+\int_{\text {Space }}\left(f\left(s, a, p^{\prime}\right)-f(s, a, p)\right)^{2} P_{s-}^{\epsilon}(p) \Pi_{s-}^{\epsilon}\left(p, d p^{\prime}\right), Z_{s}^{M, \epsilon}\right\rangle d s
\end{aligned}
$$

where

$$
\begin{aligned}
& \text { Local }_{\text {Martingale }}=-\left\langle f_{t}, Z_{t}^{M, \epsilon}\right\rangle^{2}+\left\langle f_{0}, Z_{0}^{M, \epsilon}\right\rangle^{2}+2 \int_{0}^{t}\left\langle f_{u}, Z_{u}^{M, \epsilon}\right\rangle\left\langle\left(\frac{\partial}{\partial u}+\frac{\partial}{\partial a}\right) f(u, a, p), Z_{u}^{M, \epsilon}(d a, d p)\right\rangle d u \\
& +2 \int_{0}^{t}\left\langle f_{s}, Z_{s}^{M, \epsilon}\right\rangle\left\langle(2 f(s, 0, p)-f(s, a, p)) B(a, p)+\int_{\text {Space }}\left(f\left(s, a, p^{\prime}\right)-f(s, a, p)\right) P_{s-}^{\epsilon}(p) \Pi_{s-}^{\epsilon}\left(p, d p^{\prime}\right), Z_{s}^{M, \epsilon}\right\rangle d s .
\end{aligned}
$$

Using uniqueness of Doob decomposition we find that (40) is satisfied and

$$
\mathbb{E}\left(\left\langle\mathcal{M}_{t}^{M, \epsilon}(f)\right\rangle\right) \leq \frac{T}{M} 3(1+C)\|f\|_{L^{\infty}}^{2} \mathbb{E}\left(\sup _{t \in[0, T]}\left\langle 1, Z_{t}^{M, \epsilon}(d a, d p)\right\rangle+\sup _{t \in[0, T]}\left\langle 1, Z_{t}^{M, \epsilon}(d a, d p)\right\rangle^{2}\right)<\infty .
$$

Therefore $\mathcal{M}_{t}^{M, \epsilon}(f)$ is a $L^{2}$ martingale of angle brackets process (40). We have directly (using Rolle's theorem) that $\mathcal{M}_{t}^{M, \epsilon}(f)$ and $\mathcal{V}_{t}^{M, \epsilon}(f)$ satisfy (42)-(44).

\subsubsection{Uniform bounds on $Z_{t}^{M, \epsilon}$}

Lemma 5.3 Let $q \geq 1$ and assume that

$$
\sup _{M, \epsilon} \mathbb{E}\left(\left(\iiint 1 Z_{0}^{M, \epsilon}(d a, d p)\right)^{q}\right)<\infty
$$

then we have that

$$
C_{0}^{T}=\sup _{M, \epsilon} \mathbb{E}\left(\left(\sup _{t \in[0, T]} \iiint \int 1 Z_{t}^{M, \epsilon}(d a, d p)\right)^{q}\right)<\infty, \quad \forall T>0 .
$$

Proof Using equation (35) for $F: x \mapsto x^{q}$ and $f=1$, we find that

$$
\begin{aligned}
0=\left(\left\langle 1, Z_{t}^{M, \epsilon}\right\rangle\right)^{q}- & \left(\left\langle 1, Z_{0}^{M, \epsilon}\right\rangle\right)^{q} \\
& -\int_{0}^{t} \int_{\varepsilon} 1_{n<N_{s-}^{M}}\left[\left(\left(\left\langle 1, Z_{s}^{M, \epsilon}\right\rangle+\frac{1}{M}\right)^{q}-\left\langle 1, Z_{s}^{M, \epsilon}\right\rangle^{q}\right) 1_{0 \leq \Theta<B_{n, s-}^{M, \epsilon}} Q\left(d s,(d n), d \Theta, \Pi_{s-}^{\epsilon}\left(p, d p^{\prime}\right)\right)\right] .
\end{aligned}
$$

Since $(1+y)^{q}-y^{q} \leq q 2^{q-1}\left(1+y^{q-1}\right)$, we have that

$$
\left\langle 1, Z_{t}^{M, \epsilon}\right\rangle^{q} \leq\left\langle 1, Z_{0}^{M, \epsilon}\right\rangle^{q}+q 2^{q-1} \int_{0}^{t} \int_{\varepsilon} 1_{n<N_{s-}^{M}}\left[\left(1+\left\langle 1, Z_{s}^{M, \epsilon}\right\rangle^{q-1}\right) 1_{0 \leq \Theta<B_{n, s-}^{M, \epsilon}} Q\left(d s,(d n), d \Theta, \Pi_{s-}^{\epsilon}\left(p, d p^{\prime}\right)\right)\right],
$$


$\sup _{u \leq \min \left(T_{N}^{M}, t\right)}\left\langle 1, Z_{u}^{M, \epsilon}\right\rangle^{q} \leq\left\langle 1, Z_{0}^{M, \epsilon}\right\rangle^{q}+q 2^{q-1} \int_{0}^{t} \int_{\varepsilon} 1_{n<N_{s-}^{M}}\left[\left(1+\left\langle 1, Z_{s}^{M, \epsilon}\right\rangle^{q-1}\right) 1_{0 \leq \Theta<B_{n, s-}^{M, \epsilon}} Q\left(d s,(d n), d \Theta, \Pi_{s-}^{\epsilon}\left(p, d p^{\prime}\right)\right)\right]$ and $\mathbb{E}\left(\sup _{u \leq \min \left(T_{N}^{M}, t\right)}\left\langle 1, Z_{u}^{M, \epsilon}\right\rangle^{q}\right) \leq \mathbb{E}\left(\left\langle 1, Z_{0}^{M, \epsilon}\right\rangle^{q}\right)+q 2^{q-1} \int_{0}^{t} \int_{\text {Age } \times \text { Space }} \mathbb{E}\left(\left(1+\left\langle 1, Z_{s}^{M, \epsilon}\right\rangle^{q-1}\right) Z_{s}^{M, \epsilon}\right) d s$. Using the stopping time $\tau_{N}^{M, \epsilon}$ with $\bar{N}_{t}=E\left(\sup _{s \leq \min \left(t, \tau_{N}^{M, \epsilon}\right)} N_{s}\right)$ and noticing that $x^{q}+x \leq 2(1+x)$ for all $x \geq 0$ and $q \geq 1$, we have that

$$
\mathbb{E}\left(\sup _{u \leq \min \left(T_{N}^{M}, t\right)}\left\langle 1, Z_{u}^{M, \epsilon}\right\rangle^{q}\right) \leq\left[\mathbb{E}\left(\left\langle 1, Z_{0}^{M, \epsilon}\right\rangle^{q}\right)+t q 2^{q}\right]+q 2^{q} \int_{0}^{t} \mathbb{E}\left(\sup _{u \leq \min \left(T_{N}^{M}, s\right)}\left\langle 1, Z_{u}^{M, \epsilon}\right\rangle^{q}\right) d s .
$$

Now, using by Gronwall lemma, we find that

$$
\mathbb{E}\left(\sup _{u \leq \min \left(\tau_{N}^{M, \epsilon}, t\right)}\left\langle 1, Z_{u}^{M, \epsilon}\right\rangle^{q}\right) \leq\left[\mathbb{E}\left(\left\langle 1, Z_{0}^{M, \epsilon}\right\rangle^{q}\right)+t q 2^{q}\right] e^{q 2^{q} t}
$$

Thus, $\lim _{N \rightarrow \infty} \tau_{N}^{M, \epsilon}=\infty$ for all $(M, \epsilon)$ and for all $t \leq T$

$$
C_{0}^{t}=\sup _{M, \epsilon} \mathbb{E}\left(\left(\sup _{u \leq t}\left\langle 1, Z_{u}^{M, \epsilon}\right\rangle\right)^{q}\right) \leq\left[\sup _{M, \epsilon} \mathbb{E}\left(\left\langle 1, Z_{0}^{M, \epsilon}\right\rangle^{q}\right)+t q 2^{q}\right] e^{q 2^{q} t} .
$$

Lemma 5.4 Let $m \geq 1$ and assume that (where $d p=r^{2} \sin (\theta) d r d \theta d \phi$ )

$$
\left.\sup _{M, \epsilon} \mathbb{E}\left(\iiint \int a^{m} Z_{0}^{M, \epsilon}(d a, d p)\right)\right)<\infty
$$

then we have that

$$
C_{m}^{T}=\sup _{M, \epsilon} \mathbb{E}\left(\sup _{t \in[0, T]} \iiint \int a^{m} Z_{t}^{M, \epsilon}(d a, d p)\right)<\infty, \quad \forall T>0 .
$$

Proof We have that $Z_{u}^{M, \epsilon}$ satisfies (35), for $F: x \mapsto x$ and $f(a)=a^{m}$, we find that

$$
\begin{aligned}
&\left\langle a^{m}, Z_{t}^{M, \epsilon}\right\rangle=\left\langle a^{m}, Z_{0}^{M, \epsilon}\right\rangle+m \int_{0}^{t} \iint_{\mathbb{R}_{+} \times \text {Space }} a^{m-1} Z_{u}^{M, \epsilon}(d a, d p) d u \\
& \quad-\frac{1}{M}\left[\int_{0}^{t} \int_{\varepsilon} 1_{n<N_{s-}^{M}} A_{n}^{M, \epsilon}(s-)^{m} 1_{0 \leq \Theta<B_{n, s-}^{M, \epsilon}} Q\left(d s,(d n), d \Theta, \Pi_{s-}^{\epsilon}\left(p, d p^{\prime}\right)\right)\right] \\
&\left\langle a^{m}, Z_{t}^{M, \epsilon}\right\rangle \leq\left\langle a^{m}, Z_{0}^{M, \epsilon}\right\rangle+m \int_{0}^{t} \iint_{\mathbb{R}_{+} \times S p a c e} a^{m-1} Z_{u}^{M, \epsilon}(d a, d p) d u
\end{aligned}
$$

and finally we find that

$$
\mathbb{E}\left(\left\langle a^{m}, Z_{t}^{M, \epsilon}\right\rangle\right) \leq \mathbb{E}\left(\left\langle a^{m}, Z_{0}^{M, \epsilon}\right\rangle\right)+m \int_{0}^{t} \mathbb{E}\left(\left\langle a^{m-1} Z_{u}^{M, \epsilon}(d a, d p)\right\rangle\right) d u
$$

and using that $a^{m-1} \leq a^{m}+1$ and (49), we have that

$\left.\mathbb{E}\left(\sup _{u \leq \min \left(\tau_{N}^{M, \epsilon}, t\right)}\left\langle a^{m}, Z_{u}^{M, \epsilon}\right\rangle\right) \leq \mathbb{E}\left(\left\langle a^{m}, Z_{0}^{M, \epsilon}\right\rangle\right)+m t\left[\sup _{M, \epsilon} \mathbb{E}\left(\left\langle 1, Z_{0}^{M, \epsilon}\right\rangle\right)+2 t\right] e^{2 t}\right]+m \int_{0}^{t} \mathbb{E}\left(\left\langle a^{m}, Z_{u}^{M, \epsilon}(d a, d p)\right\rangle\right) d u$ 
Using Gronwall lemma we find

$$
\mathbb{E}\left(\sup _{u \leq \min \left(\tau_{N}^{M, \epsilon}, t\right)}\left\langle a^{m}, Z_{u}^{M, \epsilon}\right\rangle\right) \leq\left(\mathbb{E}\left(\left\langle a^{m}, Z_{0}^{M, \epsilon}\right\rangle\right)+m t\left[\sup _{M, \epsilon} \mathbb{E}\left(\left\langle 1, Z_{0}^{M, \epsilon}\right\rangle\right)+2 t\right] e^{2 t}\right) e^{m t}
$$

Therefore, under assumption (50), we prove that (51) is satisfied.

Lemma 5.5 Let $m \geq 1$ and assume that (where $d p=r^{2} \sin (\theta) d r d \theta d \phi$ )

$$
\left.\left.\sup _{M, \epsilon} \mathbb{E}\left(\iiint \int r^{m} Z_{0}^{M, \epsilon}(d a, d p)\right)\right)<\infty, \quad \sup _{M, \epsilon} \mathbb{E}\left(\iiint \int 1 Z_{0}^{M, \epsilon}(d a, d p)\right)\right)<\infty,
$$

then we have that

$$
D_{m}^{T}=\sup _{M, \epsilon} \mathbb{E}\left(\sup _{t \in[0, T \epsilon]} \iiint \int r^{m} Z_{t}^{M, \epsilon}(d a, d p)\right)<\infty, \quad \forall T>0 .
$$

Proof We have that $Z_{u}^{M, \epsilon}$ satisfies (35), for $F: x \mapsto x$ and $f(a)=r^{m}$, we find that

$$
\begin{aligned}
\sup _{u \leq \min \left(\tau_{N}^{M, \epsilon}, t\right)}\left\langle r^{m}, Z_{u}^{M, \epsilon}\right\rangle=\left\langle r^{m},\right. & \left.Z_{0}^{M, \epsilon}\right\rangle+\frac{1}{M}\left[\int _ { 0 } ^ { t } \int _ { \varepsilon } 1 _ { n < N _ { s - } ^ { M } } \left[\left(X_{n}^{M, \epsilon}(s-)\right)^{m} 1_{0 \leq \Theta<B_{n, s-}^{M, \epsilon}}\right.\right. \\
& \left.\left.+\left(\left(p^{\prime}\right)^{m}-\left(X_{n}^{M, \epsilon}(s-)\right)^{m}\right) 1_{\left.B_{n, s-}^{M, \epsilon} \leq \Theta<B_{n, s-}^{M, \epsilon}+P_{s-}^{\epsilon}\right)}\right] Q\left(d s,(d n), d \Theta, \Pi_{s-}^{\epsilon}\left(p, d p^{\prime}\right)\right)\right]
\end{aligned}
$$

and finally we find that

$$
\begin{aligned}
& \mathbb{E}\left(\sup _{u \leq \min \left(\tau_{N}^{M, \epsilon}, t\right)}\left\langle r^{m}, Z_{u}^{M, \epsilon}\right\rangle\right) \leq \mathbb{E}\left(\left\langle r^{m}, Z_{0}^{M, \epsilon}\right\rangle\right) \\
& \quad+\int_{0}^{t} \mathbb{E}\left(\left\langle r^{m}, Z_{u}^{M, \epsilon}\right\rangle\right)+\left\|P_{s-}^{\epsilon}\right\| \epsilon m 2^{m}\left(2 \mathbb{E}\left(\left\langle 1, Z_{u}^{M, \epsilon}\right\rangle\right)+\mathbb{E}\left(\left\langle r^{m}, Z_{u}^{M, \epsilon}\right\rangle\right)\right) d u, \\
& \mathbb{E}\left(\sup _{u \leq \min \left(\tau_{N}^{M, \epsilon}, t\right)}\left\langle r^{m}, Z_{u}^{M, \epsilon}\right\rangle\right) \leq \mathbb{E}\left(\left\langle r^{m}, Z_{0}^{M, \epsilon}\right\rangle\right)+\int_{0}^{t} \mathbb{E}\left(\left\langle r^{m}, Z_{u}^{M, \epsilon}\right\rangle\right)\left(1+\left\|P_{s-}^{\epsilon}\right\| \epsilon m 2^{m}\right) d u+\left\|P_{s-}^{\epsilon}\right\| \epsilon m 2^{m+1} t C_{0}^{T} .
\end{aligned}
$$

Using Gronwall lemma we find that for all $t<T$ :

$$
\mathbb{E}\left(\sup _{u \leq \min \left(\tau_{N}^{M, \epsilon}, t \epsilon\right)}\left\langle r^{m}, Z_{u}^{M, \epsilon}\right\rangle\right) \leq\left(\mathbb{E}\left(\left\langle r^{m}, Z_{0}^{M, \epsilon}\right\rangle+\left\|P_{s-}^{\epsilon}\right\| \epsilon^{2} m 2^{m+1} t C_{0}^{T}\right) e^{\left(1+\left\|P_{s-}^{\epsilon}\right\| \epsilon^{2} m 2^{m}\right) t}\right.
$$

with and (53) holds.

Lemma 5.6 Let $m \geq 1$ and assume that

$$
\sup _{M, \epsilon} \mathbb{E}\left(\left(\iiint \int a^{m} Z_{0}(d a, d p)\right)^{2}\right)<\infty, \quad \sup _{M, \epsilon} \mathbb{E}\left(\left(\iiint \int a^{2 m} Z_{0}(d a, d p)\right)\right),
$$

then we have that

$$
C_{m, 2}^{T}=\sup _{M, \epsilon} \mathbb{E}\left(\left(\sup _{t \in[0, T]} \iiint \int a^{m} Z_{t}^{M, \epsilon}(d a, d p)\right)^{2}\right)<\infty, \quad \forall T>0 .
$$


Proof We have that (see lemma 5.2), for all $t \leq T$,

$$
\begin{aligned}
& \left(\left\langle a^{m}, Z_{t}^{M, \epsilon}\right\rangle\right)^{2}=\left(\left\langle a^{m}, Z_{0}^{M, \epsilon}\right\rangle\right)^{2}+2 m \int_{0}^{t}\left(\left\langle a^{m}, Z_{u}^{M, \epsilon}\right\rangle\right) \iint_{\mathbb{R}_{+} \times \text {Space }} a^{m-1} Z_{u}^{M, \epsilon}(d a, d p) d u \\
& +\int_{0}^{t} \int_{\varepsilon} 1_{n<N_{s-}^{M}}\left[\left(-\frac{2}{M} A_{n}^{M, \epsilon}(s-)^{m}\left(\left\langle a^{m}, Z_{s}^{M, \epsilon}\right\rangle\right)+\frac{1}{M^{2}} A_{n}^{M, \epsilon}(s-)^{2 m}\right) 1_{0 \leq \Theta<B_{n, s-}^{M, \epsilon}}\right] Q\left(d s,(d n), d \Theta, \Pi_{s-}^{\epsilon}\left(p, d p^{\prime}\right)\right)
\end{aligned}
$$

therefore, we find that,

$$
\begin{aligned}
\mathbb{E}\left(\sup _{u \leq \min \left(\tau_{N}^{M, \epsilon}, t\right)}\left(\left\langle a^{m}, Z_{u}^{M, \epsilon}\right\rangle\right)^{2}\right) & \leq \mathbb{E}\left(\left(\left\langle a^{m}, Z_{0}^{M, \epsilon}\right\rangle\right)^{2}\right) \\
& +2 m \int_{0}^{t} \mathbb{E}\left(\left(\left\langle a^{m}, Z_{u}^{M, \epsilon}\right\rangle\right) \iint_{\mathbb{R}_{+} \times \text {Space }} a^{m-1} Z_{u}^{M, \epsilon}(d a, d p)\right) d u+\int_{0}^{t} \frac{1}{M} \mathbb{E}\left(\left\langle a^{2 m}, Z_{u}^{M, \epsilon}\right\rangle\right) d u
\end{aligned}
$$

and using the lemma 5.4 and the Gronwall lemma (and noticing that $a^{m-1} \leq a^{m}+1$ ), (55) holds.

Lemma 5.7 Let $m \geq 1$ and assume that

$$
\sup _{M, \epsilon} \mathbb{E}\left(\left(\iiint \int r^{m} Z_{0}(d a, d p)\right)^{2}\right)<\infty, \quad \sup _{M, \epsilon} \mathbb{E}\left(\left(\iiint \int r^{2 m} Z_{0}(d a, d p)\right)\right),
$$

and (19), then we have that

$$
D_{m, 2}^{T}=\sup _{M, \epsilon} \mathbb{E}\left(\left(\sup _{t \in[0, T \epsilon]} \iiint \int r^{m} Z_{t}^{M, \epsilon}(d a, d p)\right)^{2}\right)<\infty, \quad \forall T>0
$$

The proof of this lemma is similar to the previous ones.

\subsection{Technical lemmaes : PDE}

In this part, we prove the existence, regularity and compactness of solution to the intermediate equation.

\subsubsection{Proof of existence and uniqueness of solution to (12)}

Lemma 5.8 (Existence/Uniqueness) Let $Z_{0}^{\epsilon}(a, p)=\rho_{0}^{\epsilon}(a, p)$ dadp satisfying (22) with $\rho_{0}^{\epsilon} \in C^{1}$ and

$$
\rho_{0}^{\epsilon}(a=0, p)=2 \int B(a, p) \rho_{0}^{\epsilon}(a, p) d a,
$$

then $Z_{t}^{\epsilon}(a, p)=\lim _{M_{k} \rightarrow \infty} Z_{t}^{M_{k}, \epsilon}(d a, d p)$ is equal to $\rho^{\epsilon}(t, a, p) d a d p$ with $\rho^{\epsilon} C^{1}$ solution to (12).

Proof Let $\mathcal{T}: g \mapsto f$ solution to

$$
\left\{\begin{array}{l}
\left(\frac{\partial}{\partial t}+\frac{\partial}{\partial a}\right) f-K[g](t, p)=-K_{T}(t, p) f(t, a, p), \\
f(t, 0, p)=2 \int B(a, p) f(t, a, p) d a, \quad f(t=0, ., .)=\rho_{0}^{\epsilon}(., .),
\end{array}\right.
$$

with $K[f](t, p)=\iiint \tilde{P}_{\infty}^{\epsilon}\left(p^{\prime}\right) \frac{\tilde{\Pi}_{\infty}^{\epsilon}\left(p^{\prime}, d p\right)}{d p} f\left(t, a, p^{\prime}\right) d p^{\prime}, K_{T}(t, p)=\tilde{P}_{\infty}^{\epsilon}(p)$,

$$
\tilde{P}_{\infty}^{\epsilon}\left(p^{\prime}\right)=\frac{C}{\epsilon^{2}} \sum_{i, j, k} \Psi_{i, j, k}^{\epsilon}\left(p^{\prime}\right) R\left(\frac{\iiint \Psi_{i, j, k}^{\epsilon}(p) g d a d p V o l_{G}}{V o l_{i, j, k}^{\epsilon}}\right),
$$


and

$$
\frac{\tilde{\Pi}_{\infty}^{\epsilon}\left(p^{\prime}, d p\right)}{d p}=\frac{\Psi^{\epsilon}\left(\left|p-p^{\prime}\right|\right) \tilde{G}_{s-}^{\epsilon}(p)}{\iiint \Psi^{\epsilon}\left(\left|q-p^{\prime}\right|\right) \tilde{G}_{s-}^{\epsilon}(q) d q}, \text { with } \tilde{G}_{s-}^{\epsilon}(.)=1-\sum_{i, j, k} \Psi_{i, j, k}^{\epsilon}(.) R\left(\frac{\iiint \Psi_{i, j, k}^{\epsilon}(p) g d a d p V o l_{G}}{V o l_{i, j, k}^{\epsilon}}\right)
$$

Using Gronwall lemma and $\|B\|_{\infty} \leq 1$, we have that

$$
\iiint \int f(t, a, p) d a d p \leq \iiint \int \rho_{0}^{\epsilon}(a, p) d a d p e^{2 t}
$$

By computation, and using that $\left|R^{\prime}\right| \leq \frac{1}{\sigma}$, we find that

$$
|K[g]-K[\tilde{g}]|(t, p) \leq \frac{3 V o l_{G}}{\sigma\left(1-R\left(\iiint \int \rho_{0}^{\epsilon}(a, p) d a d p e^{2 t}\right)\right) V o l_{i, j, k}^{\epsilon}} \iiint \int|g-\tilde{g}| d a d p .
$$

Therefore, using the Characteristics (in $(a, t)$ ) of the transport equation (57) (see [20]), we have existence and uniqueness of the solution. Moreover, for $\phi: t \mapsto \int_{t}^{\infty} 2 e^{-\frac{C}{\epsilon^{2}}(s-t)} d s$, and $f=\mathcal{T}(g), \tilde{f}=\mathcal{T}(\tilde{g})$, we have that (multiplying (57) by $\phi$ and integrating in $(a, p)$ )

$$
\frac{d}{d t} \iiint \int|f-\tilde{f}| \phi(t) d a d p \leq \frac{3 V o l_{G}}{\sigma\left(1-R\left(\iiint \int \rho_{0}^{\epsilon}(a, p) d a d p e^{2 t}\right)\right) V o l_{i, j, k}^{\epsilon}} \iiint \int|g-\tilde{g}| \phi(t) d a d p,
$$

and so

$$
\iiint \int|f-\tilde{f}| \phi(t) d a d p \leq \int_{0}^{t} \frac{3 V_{0} l_{G}}{\sigma\left(1-R\left(\iiint \int \rho_{0}^{\epsilon}(a, p) d a d p e^{2 s}\right)\right) V_{o l}^{\epsilon} l_{, k}} \iiint \int|g-\tilde{g}| \phi(s) d a d p d s .
$$

Therefore $\mathcal{T}: g \in E \mapsto f \in E$ with $E=C\left([0, T], L^{1}\left(\mathbb{R}_{+} \times \mathbb{R}^{3}\right)\right)$, is a contracting mapping for $T>0$ small enough and there exists an unique solution to (12). Now, we construct a solution on $[T, 2 T] \ldots$ and finally on $\mathbb{R}_{+}$. Since $\Psi_{\epsilon}$ and $R$ are $C^{\infty}$, the regularity of $\rho^{\epsilon}$, solution to the transport equation (12) with the boundary condition 56 , is given by those of $\rho_{0}^{\epsilon}$ (under the assumption $(56)$, see $[20,18,11,27]$ ). Using uniqueness, we find that $Z_{t}^{\epsilon}(a, p)=\rho^{\epsilon}(t, a, p) d a d p$.

\subsubsection{Compactness of $M_{\epsilon}^{1}(t, p):=\int \rho^{\epsilon}(t, a, p) d a$}

Lemma 5.9 Let $T>0, \rho^{\epsilon}$ solution to (12) and assume that (20) is satisfied then we can extract a convergent subsequence of $M_{\epsilon}^{1}(t, p):=\int \rho^{\epsilon}(t, a, p) d a$ in $L^{2}([0, T] \times$ Space $)$.

Proof Since $M_{\epsilon}^{1}(0, p) \in L^{2}$ (Space) by assumption (20), we have, by integrating equation (12) that $M_{\epsilon}^{1}(t, p):=$ $\int \rho^{\epsilon}(t, a, p) d a$ follows the equation

$$
\left\{\begin{array}{l}
\frac{\partial}{\partial t} M_{\epsilon}^{1}(t, p)-\iiint P_{\infty}^{\epsilon}\left(p^{\prime}\right) \frac{\Pi_{\infty}^{\epsilon}\left(p^{\prime}, d p\right)}{d p} M_{\epsilon}^{1}\left(t, p^{\prime}\right) d p^{\prime}+P_{\infty}^{\epsilon}(p) M_{\epsilon}^{1}(t, p)=2 \int B(a, p) \rho^{\epsilon}(t, a, p) d a, \\
M_{\epsilon}^{1}(t=0, p)=\int \rho_{0}^{\epsilon}(a, p) d a .
\end{array}\right.
$$

First integrating (58) with respect to $p$ and Gronwall lemma, we have that

$$
\int M_{\epsilon}^{1}(0, p) d p \leq \int M_{\epsilon}^{1}(t, p) d p \leq \int M_{\epsilon}^{1}(0, p) d p e^{2 T}, \quad \forall t \in[0, T],
$$

therefore $\rho^{\epsilon}$ is uniformly bounded in $L^{\infty}\left([0, T], L^{1}(\right.$ Age $\times$ Space $\left.)\right)$. Secondly, multiplying (58) by $M_{\epsilon}^{1}$ and integrating with respect to $p$ we find that 


$$
\begin{array}{r}
\frac{\partial}{\partial t} \frac{1}{2} \int\left(M_{\epsilon}^{1}(t, p)\right)^{2} d p=\iint\left[\frac{P_{\infty}^{\epsilon}\left(p^{\prime}\right) M_{\epsilon}^{1}\left(t, p^{\prime}\right)}{\iiint \Psi^{\epsilon}\left(\left|q-p^{\prime}\right|\right) G_{s-}^{\epsilon}(q) d q} G_{s-}^{\epsilon}(p)\right]\left(M_{\epsilon}^{1}(t, p)-M_{\epsilon}^{1}\left(t, p^{\prime}\right)\right) \Psi^{\epsilon}\left(\left|p^{\prime}-p\right|\right) d p d p^{\prime} \\
+\iint B(a, p) \rho^{\epsilon}(t, a, p) d a M_{\epsilon}^{1}(t, p) d p
\end{array}
$$

that can be rewritten as,

$$
\begin{aligned}
\frac{\partial}{\partial t} \frac{1}{2} \int\left(M_{\epsilon}^{1}(t, p)\right)^{2} d p=\frac{1}{2} \iint\left[\frac{P_{\infty}^{\epsilon}\left(p^{\prime}\right) M_{\epsilon}^{1}\left(t, p^{\prime}\right)}{\iiint \Psi^{\epsilon}\left(\left|q-p^{\prime}\right|\right) G_{s-}^{\epsilon}(q) d q} G_{s-}^{\epsilon}(p)-\frac{P_{\infty}^{\epsilon}(p) M_{\epsilon}^{1}(t, p)}{\iiint \Psi^{\epsilon}(|q-p|) G_{s-}^{\epsilon}(q) d q} G_{s-}^{\epsilon}\left(p^{\prime}\right)\right] \\
\left(M_{\epsilon}^{1}(t, p)-M_{\epsilon}^{1}\left(t, p^{\prime}\right)\right) \Psi^{\epsilon}\left(\left|p^{\prime}-p\right|\right) d p d p^{\prime}+\iint B(a, p) \rho^{\epsilon}(t, a, p) d a M_{\epsilon}^{1}(t, p) d p .
\end{aligned}
$$

Let $p_{+}=\left(p+p^{\prime}\right) / 2$ and $p_{-}=\left(p-p^{\prime}\right) / 2$, then we have $p=p_{+}+p_{-}, p^{\prime}=p_{+}-p_{-}$and for all $A, B C^{1}-$ functions we have that

$$
\begin{aligned}
A\left(p^{\prime}\right) B(p)-A(p) B\left(p^{\prime}\right)= & A\left(p_{+}-p_{-}\right) B\left(p_{+}+p_{-}\right)-B\left(p_{+}-p_{-}\right) A\left(p_{+}+p_{-}\right) \\
= & \left(A\left(p_{+}\right)-\nabla A\left(p_{+}\right) p_{-}+o\left(p_{-}\right)\right)\left(B\left(p_{+}\right)+\nabla B\left(p_{+}\right) p_{-}+o\left(p_{-}\right)\right) \\
& -\left(A\left(p_{+}\right)+\nabla A\left(p_{+}\right) p_{-}+o\left(p_{-}\right)\right)\left(B\left(p_{+}\right)-\nabla B\left(p_{+}\right) p_{-}+o\left(p_{-}\right)\right) \\
= & 2\left(A\left(p_{+}\right) \nabla B\left(p_{+}\right) p_{-}-B\left(p_{+}\right) \nabla A\left(p_{+}\right) p_{-}\right)+o\left(p_{-}\right) .
\end{aligned}
$$

Noticing that $M_{\epsilon}^{1}(t, p)-M_{\epsilon}^{1}\left(t, p^{\prime}\right)=M_{\epsilon}^{1}\left(t, p_{+}+p_{-}\right)-M_{\epsilon}^{1}\left(t, p_{+}-p_{-}\right)=2 \nabla M_{\epsilon}^{1}\left(t, p_{+}\right) p_{-}+o\left(p_{-}\right)$and changing the variables in the integral, we find that

$$
\begin{aligned}
\frac{\partial}{\partial t} \frac{1}{2} \int\left(M_{\epsilon}^{1}(t, p)\right)^{2} d p=4 \iint[ & \left(\frac{P_{\infty}^{\epsilon} M_{\epsilon}^{1}(t, .)}{\iiint \Psi^{\epsilon}(|q-.|) G_{s-}^{\epsilon}(q) d q}\left(p_{+}\right) \nabla G_{s-}^{\epsilon}\left(p_{+}\right) 2 p_{-}\right. \\
& \left.-G_{s-}^{\epsilon}\left(p_{+}\right) \nabla \frac{P_{\infty}^{\epsilon} M_{\epsilon}^{1}(t, .)}{\iiint \Psi^{\epsilon}(|q-.|) G_{s-}^{\epsilon}(q) d q}\left(p_{+}\right) 2 p_{-}\right] \\
( & \left.2 \nabla M_{\epsilon}^{1}\left(t, p_{+}\right) p_{-}\right) \Psi^{\epsilon}\left(2\left|p_{-}\right|\right) d p_{+} d p_{-}+\iint B(a, p) \rho^{\epsilon}(t, a, p) d a M_{\epsilon}^{1}(t, p) d p+o(1) .
\end{aligned}
$$

Using that

$$
P_{\infty}^{\epsilon}\left(p^{\prime}\right)=\frac{C}{\epsilon^{2}} \sum_{i, j, k} \Psi_{i, j, k}^{\epsilon}\left(p^{\prime}\right) R\left(\frac{\iiint \Psi_{i, j, k}^{\epsilon}(p) M_{\epsilon}^{1}(s, p) d p V o l_{G}}{V o l_{i, j, k}^{\epsilon}}\right), \text { with } R(x)=\frac{1}{1+e^{-\frac{x-\mu}{\sigma}}}
$$

$$
\Pi_{\infty}^{\epsilon}\left(p^{\prime}, d p\right)=\frac{\Psi^{\epsilon}\left(\left|p^{\prime}-p\right|\right) G_{s-}^{\epsilon}(p) d p}{\iiint \Psi^{\epsilon}\left(\left|q-p^{\prime}\right|\right) G_{s-}^{\epsilon}(q) d q}, \text { with } G_{s-}^{\epsilon}(.)=1-\sum_{i, j, k} \Psi_{i, j, k}^{\epsilon}(.) R\left(\frac{\iiint \Psi_{i, j, k}^{\epsilon}(p) M_{\epsilon}^{1}(s, p) d p V o l_{G}}{V o l_{i, j, k}^{\epsilon}}\right)
$$

we find that

$$
\begin{array}{r}
\frac{\partial}{\partial t} \frac{1}{2} \int\left(M_{\epsilon}^{1}(t, p)\right)^{2} d p=4 C \iint\left[M_{\epsilon}^{1}(t, .) \frac{R}{1-R}\left(M_{\epsilon}^{1}(t, .)\right)(1-R)^{\prime} \nabla M_{\epsilon}^{1}\left(t, p_{+}\right) 2 \frac{p_{-}}{\epsilon}\right. \\
\left.-M_{\epsilon}^{1}\left(t, p_{+}\right)(1-R)\left(\frac{R}{1-R}\right)^{\prime}\left(M_{\epsilon}^{1}\left(t, p_{+}\right)\right) \nabla M_{\epsilon}^{1}\left(t, p_{+}\right) 2 \frac{p_{-}}{\epsilon}-R\left(M_{\epsilon}^{1}\left(t, p_{+}\right)\right) \nabla M_{\epsilon}^{1}\left(t, p_{+}\right) 2 \frac{p_{-}}{\epsilon}\right] \\
\left(2 \nabla M_{\epsilon}^{1}\left(t, p_{+}\right) \frac{p_{-}}{\epsilon}\right) \Psi^{\epsilon}\left(2\left|p_{-}\right|\right) d p_{+} d p_{-}+\iint B(a, p) \rho^{\epsilon}(t, a, p) d a M_{\epsilon}^{1}(t, p) d p+o(1) .
\end{array}
$$


and

$$
\begin{aligned}
& \frac{\partial}{\partial t} \frac{1}{2} \int\left(M_{\epsilon}^{1}(t, p)\right)^{2} d p= \\
& -4 C \int\left[1+\frac{2 M_{\epsilon}^{1}\left(t, p_{+}\right)}{\sigma}\right] R\left(M_{\epsilon}^{1}\left(t, p_{+}\right)\right)\left(\nabla M_{\epsilon}^{1}\left(t, p_{+}\right) \int 2 \frac{p_{-}}{\epsilon} \Psi^{\epsilon}\left(2\left|p_{-}\right|\right) 2 \frac{{ }^{t} p_{-}}{\epsilon} d p_{-}{ }^{t} \nabla M_{\epsilon}^{1}\left(t, p_{+}\right)\right) \Psi^{\epsilon}\left(2\left|p_{-}\right|\right) d p_{+} \\
& +\iint B(a, p) \rho^{\epsilon}(t, a, p) d a M_{\epsilon}^{1}(t, p) d p+o(1) .
\end{aligned}
$$

Therefore, we have the following bound

$$
\frac{\partial}{\partial t} \frac{1}{2} \int\left(M_{\epsilon}^{1}(t, p)\right)^{2} d p+4 C R(0) \lambda_{m} \iint\left[1+\frac{2 M_{\epsilon}^{1}\left(t, p_{+}\right)}{\sigma}\right]\left\|\nabla M_{\epsilon}^{1}(t, .)\right\|^{2}\left(t, p_{+}\right) d p_{-} \leq \int\left(M_{\epsilon}^{1}(t, p)\right)^{2} d p,
$$

with $\lambda_{m}>0$ the infimum (with respect of $\epsilon$ ) of the minimum of the eigenvalues of the definite positive matrix $\int 2 \frac{p_{-}}{\epsilon} \Psi^{\epsilon}\left(2\left|p_{-}\right|\right) 2 \frac{{ }^{t} p_{-}}{\epsilon} d p_{-}$. Finally, using Gronwall inequality we have that for all $T>0$

$$
\int\left(M_{\epsilon}^{1}(t, p)\right)^{2} d p \leq \int\left(M_{\epsilon}^{1}(0, p)\right)^{2} d p e^{2 T}, \quad \forall t \leq T
$$

and integrating the last inequality, we found that

$$
\int_{[0, T]} \iint\left[1+\frac{2 M_{\epsilon}^{1}\left(t, p_{+}\right)}{\sigma}\right]\left\|\nabla M_{\epsilon}^{1}(t, .)\right\|^{2}\left(t, p_{+}\right) d p_{-} \leq \frac{\int\left(M_{\epsilon}^{1}(0, p)\right)^{2} d p}{4 C R(0) \lambda_{m}} e^{2 T}(T+1) .
$$

We notice that, for all $T>0$, the same computation leads to (multiplying (58) by $\left(p M_{\epsilon}^{1}\right)^{2}$ and integrating with respect to $p$ )

$$
\int p\left(M_{\epsilon}^{1}(t, p)\right)^{2} d p \leq\left[\int p\left(M_{\epsilon}^{1}(0, p)\right)^{2} d p+T\left\|M_{\epsilon}^{1}\right\|_{C\left([0, T], H^{1}(\text { Space })\right)} \lambda_{M}\right] e^{2 T}, \quad \forall t \leq T,
$$

with $\lambda_{M}>0$ the supremum (with respect of $\epsilon$ ) of the maximum of the eigenvalues of the definite positive matrix $\int 2 \frac{p_{-}}{\epsilon} \Psi^{\epsilon}\left(2\left|p_{-}\right|\right) 2 \frac{{ }^{t} p_{-}}{\epsilon} d p_{-}$. Thus, we have that $\left(M_{\epsilon}^{1}\right)_{\epsilon}$ is uniformly bounded in $L^{2}\left([0, T], H^{1}(\right.$ Space $) \times L_{p d p}^{2}($ Space $\left.)\right)$. Moreover, we have directly that $\left|\frac{\partial}{\partial t} \frac{1}{2} \int\left(M_{\epsilon}^{1}(t, p)\right)^{2} d p\right|$ is uniformly bounded in $L^{2}([0, T])$. Therefore, by the (Lions-Aubin) result (see [14]), we can extract a convergent subsequence of $\left(M_{\epsilon}^{1}\right)_{\epsilon}$ in $L^{2}([0, T] \times($ Space $))$.

Lemma 5.10 Let $T>0$. Assume that $\left(\nabla M_{\epsilon}^{1}(t, .)\right)_{\epsilon}$ is $L^{\infty}\left([0, T] ; L^{2}(\right.$ Space $\left.)\right)$ and $M_{\epsilon}^{1}$ converges $C^{0}\left([0, T] ; L^{2}(S p a c e)\right)$ to $M^{1}$ as $\epsilon$ to 0 then

$$
\sum_{i, j, k} \Psi_{i, j, k}^{\epsilon}\left(p^{\prime}\right) R\left(\frac{\iiint \Psi_{i, j, k}^{\epsilon}(p) M_{\epsilon}^{1}(t, .) d a d p V o l_{G}}{V o l_{i, j, k}^{\epsilon}}\right) \rightarrow_{C^{0}\left([0, T] ; L^{2}(\text { Space })\right)} R\left(\frac{V o l_{G}\left(3 r^{2}+6 r+3\right)}{3 r^{2}+6 r r_{O}(s)+3 r_{O}(s)^{2}} M^{1}(t, p)\right) .
$$

Proof Indeed, we have that

$$
\begin{array}{r}
\int\left|\sum_{i, j, k} \Psi_{i, j, k}^{\epsilon}\left(p^{\prime}\right) R\left(\frac{\iiint \Psi_{i, j, k}^{\epsilon}(p) M_{\epsilon}^{1}(t, .) d a d p V o l_{G}}{V o l_{i, j, k}^{\epsilon}}\right)-R\left(\frac{\operatorname{Vol}_{G}\left(3 r^{2}+6 r+3\right)}{3 r^{2}+6 r r_{O}(s)+3 r_{O}(s)^{2}} M_{\epsilon}^{1}(t, p)\right)\right|^{2} d p \\
\leq\left\|R^{\prime}\right\|_{\infty}^{2} \int\left(\int_{u \leq 1}\left|M_{\epsilon}^{1}(p+\epsilon u)-M_{\epsilon}^{1}(p)\right| d u\right)^{2} d p \leq \epsilon^{2} \int\left(\int_{u \leq 1} \int_{s \in[0,1]}\left|\nabla M_{\epsilon}^{1}(p+\epsilon s u)\right| d u d s\right)^{2} d p \\
\leq \epsilon^{2} \int_{u \leq 1} \int_{s \in[0,1]}\left(\int\left|\nabla M_{\epsilon}^{1}(p+\epsilon s u)\right|^{2} d p\right) d u d s
\end{array}
$$


and

$$
\begin{aligned}
\int\left(R\left(\frac{V_{o l}\left(3 r^{2}+6 r+3\right)}{3 r^{2}+6 r r_{O}(s)+3 r_{O}(s)^{2}} M^{1}(t, p)\right)-R\left(\frac{V o l_{G}\left(3 r^{2}+6 r+3\right)}{3 r^{2}+6 r r_{O}(s)+3 r_{O}(s)^{2}}\right.\right. & \left.\left.M_{\epsilon}^{1}(t, p)\right)\right)^{2} d p \\
& \leq c s t\left\|R^{\prime}\right\|_{\infty}^{2} \int\left(M^{1}(t, p)-M_{\epsilon}^{1}(t, p)\right)^{2} d p
\end{aligned}
$$

Under the uniform boundness of $\left.\nabla M_{\epsilon}^{1}(t,).\right)_{\epsilon}$ and the $C^{0}\left([0, T] ; L^{2}(\right.$ Space $\left.)\right)$ convergence of $M_{\epsilon}^{1}$ we have that $\sum_{i, j, k} \Psi_{i, j, k}^{\epsilon}\left(p^{\prime}\right) R\left(\frac{\iiint \Psi_{i, j, k}^{\epsilon}(p) M_{\epsilon}^{1}(t, .) d a d p V o l_{G}}{V o l_{i, j, k}^{\epsilon}}\right)$ converges (as $\left.\epsilon \rightarrow 0\right)$ to $R\left(M^{1}(t, p)\right)$ in $C^{0}\left([0, T] ; L^{2}(\right.$ Space $\left.)\right)$.

Lemma 5.11 Let $T>0, \rho^{\epsilon}$ solution to (12) and assume that (20) is satisfied then we can extract a convergent subsequence of $M_{\epsilon}^{1}(t, p):=\int \rho^{\epsilon}(t, a, p) d a$ which limit is a weak solution to

$$
\begin{gathered}
\frac{\partial}{\partial t} M^{1}(t, p)+\operatorname{div}\left(M^{1}(t, p) C h(p) R \nabla(\log (1-R))\right)=H, \\
M^{1}(0, p)=\int \rho_{0}^{\epsilon}(a, p) d a
\end{gathered}
$$

and $H \in C\left([0, T], H^{1}(\right.$ Space $\left.)\right)$ and $R$ defined in (15).

Proof Let $f \in C_{c}^{1}\left(\mathbb{R}_{+} \times\right.$Space), multiplying (12) by $f$ and integrating with respect to $a$ we find that:

$$
\frac{\partial}{\partial t} \int_{p} f(p) M_{\epsilon}^{1}(t, p) d p-\iint B(a, p) f(p) \rho^{\epsilon}(t, a, p) d a d p=\iiint_{p, p^{\prime}}\left(f\left(p^{\prime}\right)-f(p)\right) \Pi_{s-}^{\epsilon}\left(p, d p^{\prime}\right) P_{s-}^{\epsilon}(p) M_{\epsilon}^{1}(t, p) d p .
$$

Rewritting the second member as follows, we have that

$$
\begin{gathered}
\iiint_{p, p^{\prime}}\left(f\left(p^{\prime}\right)-f(p)\right) \Pi_{s-}^{\epsilon}\left(p, d p^{\prime}\right) P_{s-}^{\epsilon}(p) M_{\epsilon}^{1}(t, p) d p \\
=\iiint_{p, p^{\prime}} C^{\epsilon}\left(f\left(p^{\prime}\right)-f(p)\right) \frac{\Psi^{\epsilon}\left(\left|p^{\prime}-p\right|\right) G_{s-}^{\epsilon}\left(p^{\prime}\right) d p^{\prime}}{\iiint \Psi^{\epsilon}(|q-p|) G_{s-}^{\epsilon}(q) d q} \frac{P_{s-}^{\epsilon}(p)}{C^{\epsilon}} M_{\epsilon}^{1}(t, p) d p= \\
=\iiint_{p}{ }^{t} \nabla f(p) \frac{C^{\epsilon} \iiint_{q} \Psi^{\epsilon}(|q-p|)(q-p) G_{s-}^{\epsilon}(q) d q}{\iiint \Psi^{\epsilon}(|q-p|) G_{s-}^{\epsilon}(q) d q} \frac{P_{s-}^{\epsilon}(p)}{C^{\epsilon}} M_{\epsilon}^{1}(t, p) d p+o(1) \\
=\iiint_{p}{ }^{t} \nabla f(p) \frac{C^{\epsilon} \iiint_{q} \Psi^{\epsilon}(|q-p|)(q-p){ }^{t}(q-p) d q}{\iiint \Psi^{\epsilon}(|q-p|) d q} \frac{P_{s-}^{\epsilon}(p)}{C^{\epsilon}} M_{\epsilon}^{1}(t, p) \frac{\nabla G_{s-}^{\epsilon}}{G_{s-}^{\epsilon}}(p) d p+o(1) \\
=\iiint_{p} \nabla f(p) M_{\epsilon}^{1}(t, p) C h(p) R_{\epsilon}^{t} \nabla\left(\log \left(1-R_{\epsilon}\right)\right) d p+o(1)
\end{gathered}
$$

Since, $M_{\epsilon}^{1}(t, p)$ is bounded $C\left([0, T], H^{1}(\right.$ Space $\left.)\right)$, we have that $\log \left(1-R_{\epsilon}\right) \rightarrow^{\text {Distribution }} \log (1-R)$ and by Banach Aologlu in $L^{2}$ (and identifying the limit) $\nabla \log \left(1-R_{\epsilon}\right) \rightarrow^{*-\text { weak } L^{2}} \nabla \log (1-R)$. Now, by lemmaes 5.9 and 5.10, we have that $\nabla f(p) M_{\epsilon}^{1}(t, p) C h(p) R_{\epsilon} \rightarrow{ }^{2} \nabla f(p) M^{1}(t, p) C h(p) R$ and so (there exists $H \in C\left([0, T], L^{2}\right)$, the limit of $\iint B(a, p) f(p) \rho^{\epsilon}(t, a, p) d a d p$ as $\left.\epsilon \rightarrow 0\right)$

$$
\frac{\partial}{\partial t} \int_{p} f(p) M^{1}(t, p) d p-\iint f(p) H(t, p) d p=\iiint_{p} \nabla f(p) M^{1}(t, p) C h(p) R^{t} \nabla(\log (1-R)) d p .
$$

Therefore the limit is $C\left([0, T], H^{1}\right)$ and is a weak solution to

$$
\frac{\partial}{\partial t} M^{1}(t, p)+\operatorname{div}\left(M^{1}(t, p) C h(p) R \nabla(\log (1-R))\right)=H,
$$

with $M^{1}(0, p)=\int \rho_{0}(a, p) d a$. 


\subsubsection{Proof of existence and uniqueness of solution to (15)bis}

Lemma 5.12 Let $T>0, \rho^{\epsilon}$ solution to (12) and assume that (20) is satisfied then we can extract a convergent subsequence of $\left(\rho^{\epsilon}\right)_{\epsilon}$ in $L^{2}([0, T] \times$ Space $\times$ Age $)$.

Proof Differentiate (12) with respect to $a$ leads to

$$
\left\{\begin{array}{l}
\left(\frac{\partial}{\partial t}+\frac{\partial}{\partial a}\right) \frac{\partial}{\partial a} \rho^{\epsilon}-\iiint P_{\infty}^{\epsilon}\left(p^{\prime}\right) \frac{\Pi_{\infty}^{\epsilon}\left(p^{\prime}, d p\right)}{d p} \frac{\partial}{\partial a} \rho^{\epsilon}\left(t, a, p^{\prime}\right) d p^{\prime}+P_{\infty}^{\epsilon}(p) \frac{\partial}{\partial a} \rho^{\epsilon}(t, a, p)=0 \\
\left.\rho^{\epsilon}\right|_{a=0}=2 \int B(a, p) \rho^{\epsilon}(t, a, p) d a, \quad \rho_{t=0}^{\epsilon}=\rho_{0}^{\epsilon} .
\end{array}\right.
$$

Therefore, we have that

$$
\left(\frac{\partial}{\partial t}+\frac{\partial}{\partial a}\right) \int\left|\frac{\partial}{\partial a} \rho^{\epsilon}\right| d p \leq 0
$$

and for all $t, a$ and $\epsilon$, we find that

$$
\frac{\partial}{\partial t} \iint\left|\frac{\partial}{\partial a} \rho^{\epsilon}\right| d p d a \leq \int\left|\frac{\partial}{\partial a} \rho^{\epsilon}\right|(a=0) d p
$$

Now, using the intermediate equation, we find that

$$
\begin{aligned}
& \frac{\partial}{\partial a} \rho^{\epsilon}(a=0)=-2 \int B(a, p) \frac{\partial}{\partial t} \rho^{\epsilon}(t, a, p) d a \\
& +2 \iiint P_{\infty}^{\epsilon}\left(p^{\prime}\right) \frac{\Pi_{\infty}^{\epsilon}\left(p^{\prime}, d p\right)}{d p} \int B\left(a, p^{\prime}\right) \rho^{\epsilon}\left(t, a, p^{\prime}\right) d a d p^{\prime}-2 P_{\infty}^{\epsilon}(p) \int B(a, p) \rho^{\epsilon}(t, a, p) d a, \\
& \frac{\partial}{\partial a} \rho^{\epsilon}(a=0)=-2 \int B(a, p)\left[-\frac{\partial}{\partial a} \rho^{\epsilon}+\iiint P_{\infty}^{\epsilon}\left(p^{\prime}\right) \frac{\Pi_{\infty}^{\epsilon}\left(p^{\prime}, d p\right)}{d p} \rho^{\epsilon}\left(t, a, p^{\prime}\right) d p^{\prime}-P_{\infty}^{\epsilon}(p) \rho^{\epsilon}(t, a, p)\right] d a \\
& +2 \iiint P_{\infty}^{\epsilon}\left(p^{\prime}\right) \frac{\Pi_{\infty}^{\epsilon}\left(p^{\prime}, d p\right)}{d p} \int B\left(a, p^{\prime}\right) \rho^{\epsilon}\left(t, a, p^{\prime}\right) d a d p^{\prime}-2 P_{\infty}^{\epsilon}(p) \int B(a, p) \rho^{\epsilon}(t, a, p) d a, \\
& \frac{\partial}{\partial a} \rho^{\epsilon}(a=0)=-2 \int \rho^{\epsilon} \frac{\partial}{\partial a} B(a, p) d a-2 \int B(a, p)\left[\iiint P_{\infty}^{\epsilon}\left(p^{\prime}\right) \frac{\Pi_{\infty}^{\epsilon}\left(p^{\prime}, d p\right)}{d p} \rho^{\epsilon}\left(t, a, p^{\prime}\right) d p^{\prime}-P_{\infty}^{\epsilon}(p) \rho^{\epsilon}(t, a, p)\right] d a \\
& +2 \iiint P_{\infty}^{\epsilon}\left(p^{\prime}\right) \frac{\Pi_{\infty}^{\epsilon}\left(p^{\prime}, d p\right)}{d p} \int B\left(a, p^{\prime}\right) \rho^{\epsilon}\left(t, a, p^{\prime}\right) d a d p^{\prime}-P_{\infty}^{\epsilon}(p) \int B(a, p) \rho^{\epsilon}(t, a, p) d a, \text { andfinally } \\
& \frac{\partial}{\partial a} \rho^{\epsilon}(a=0)=-2 \int \rho^{\epsilon} \frac{\partial}{\partial a} B(a, p) d a-2 \iiint P_{\infty}^{\epsilon}\left(p^{\prime}\right) \frac{\Pi_{\infty}^{\epsilon}\left(p^{\prime}, d p\right)}{d p} \int\left(B(a, p)-B\left(a, p^{\prime}\right)\right) \rho^{\epsilon}\left(t, a, p^{\prime}\right) d a d p^{\prime} .
\end{aligned}
$$

Therefore, using the same computation as lemma 5.9, we have that

$$
\left.\left\|\frac{\partial}{\partial a} \rho^{\epsilon}(a=0)\right\|_{L^{2}(\text { Space })} \leq 8\|B\|_{W^{1, \infty}}\right)\left(1+\lambda_{M}^{2}\right) \sup _{\epsilon}\left\|M_{\epsilon}^{1}\right\|_{C\left([0, T], H^{1}(\text { Space })\right.}
$$

with $\lambda_{M}>0$ the sup (with respect of $\epsilon$ ) of the maximum of the eigenvalues of the definite positive matrix $\int 2 \frac{p_{-}}{\epsilon} \Psi^{\epsilon}\left(2\left|p_{-}\right|\right) 2 \frac{{ }^{t} p_{-}}{\epsilon} d p_{-}$. And so, using the same computation as lemma 5.9 , we have that

$$
\rho^{\epsilon} \in C\left([0, T], H^{1}(\text { Age } \times \text { Space }) .\right.
$$

Therefore, by the (Lions-Aubin) result (see [14]), we can extract a convergent subsequence of $\left(\rho^{\epsilon}\right)_{\epsilon}$ in $L^{2}([0, T] \times$ (Space)). 


\section{References}

[1] Aldous D., Stopping times and tightness. Ann. Probab., 6, 335-340, 1978.

[2] Brezis H., Functional Analysis, Sobolev Spaces and Partial Differential Equations, Springer Science 83 Business Media, 2010

[3] Champagnat N. , Ferrière R., and Méléard S. , Individual-based probabilistic models of adaptive evolution and various scaling approximations, In Proceedings of the 5th seminar on Stochastic Analysis, Random Fields and Applications, Probability in Progress Series, Ascona, Suisse. Birkhauser.

[4] Ferrière R. and Tran V.C. , Stochastic and deterministic models for age-structured populations with genetically variable traits, ESAIM: Proceedings, 27, 289-310, 2009.

[5] Clément F. , Michel P., Monniaux D. and Stiehl T. , Coupled somatic cell kinetics and germ cell growth: multiscale model-based insight on ovarian follicular development, SIAM : Multiscale Model. Simul., 11(3), p 719-746., 2013.

[6] Clément F. , Monniaux D. . Multiscale modelling of ovarian follicle selection. Progress in Biophysics and Molecular Biology, 113(3), 398-408, 2013

[7] Echenim N. , Monniaux D. , Sorine M. , Clément F. . Multi-scale modeling of the follicle selection process in the ovary. Mathematical Biosciences , 198, 57-79, 2005.

[8] Etheridge A., An Introduction to Superprocesses, University Lecture Series, Amer. Math. Soc., Providence, $R I, 20,2000$.

[9] Ethier S. N. and Kurtz T.G., Markov Processes: Characterization and Convergence, WILEY, ISBN: 9780-471-76986-6, 2005.

[10] Hwang H.J., Kang K., and Stevens A., Global Solutions of Nonlinear Transport Equations for Chemosensitive Movement, SIAM J. Math. Analysis, 36(4), 1177-1199, 2005.

[11] Iannelli M., Mathematical Theory of Age-Structured Population Dynamics. Applied Math. Monographs, CNR, Giardini Editori e Stampatori in Pisa, 1995.

[12] Keller E. F. and Segel L. A., Initiation of slide mold aggregation viewed as an instability, J. Theoret. Biol., 26, 399415, 1970.

[13] Keller E. F. and Segel L. A. , Model for chemotaxis, J. Theoret. Biol., 30, 225234, 1971.

[14] Lions J.-L., Equations differentielles operationnelles et problemes aux limites, Die Grundlehren der mathematischen Wissenschaften, Bd. 111, Springer-Verlag, Berlin, 1961.

[15] Méléard S., Convergence of the fluctuations for interacting diffusions with jumps associated with boltzmann equations, Stochastics and Stochastics Reports, 63, 195225, 1998.

[16] Méléard S. ; Roelly S. Sur les convergences troite ou vague de processus valeurs mesures = On the vague and weak convergence of measure-valued processes, Comptes rendus de l'Acadmie des sciences. Srie 1, Mathmatique, 317(8), 785-788, 1993.

[17] Méléard S. and Tran V.C., Trait substitution sequence process and canonical equation for age-structured populations, Journal of Mathematical Biology, 58(6), 881-921, 2009.

[18] Metz J.A.J. and Diekmann O., The dynamics of physiologically structured populations. L.N. in biomathematics, 68, Springer, 1986. 
[19] Monniaux D. , Michel P. , Postel M. , Clément F., Multiscale modeling of ovarian follicular development: from follicular morphogenesis to selection for ovulation. Biology of the Cell, ehead of pub.

[20] Perthame B., Transport Equations in Biology, Birkhuser, Frontiers in Mathematics, 2007

[21] P.-L. Lions, B. Perthame, and E. Tadmor, A kinetic formulation of multidimensional scalar conservation laws and related equations, J. Amer. Math. Soc. 7 (1), 169191, 1994.

[22] Perthame B., Kinetic formulation of conservation laws, Oxford Lecture Series in Mathematics and its Applications, 21, Oxford University Press, Oxford, 2002.

[23] Rebolledo R., Sur l'existence de solutions certains problemes de semimartingales, C. R. Acad. Sci. Paris $A B, \mathbf{2 9 0 ( 1 8 ) , ~ A 8 4 3 ~ A 8 4 6 , ~} 1980$.

[24] Roelly S., A criterion of convergence of measure-valued processes: Application to measure branching processes. Stochastics,17 , 43-65, 1986.

[25] Tran V.C., Large population limit and time behaviour of a stochastic particle model describing an agestructured population, ESAIM: PS, Vol. 12, 345-386, 2008.

[26] Tran V.C., Modles particulaires stochastiques pour des problmes d'volution adaptative et pour l'approximation de solutions statistiques. Ph.D. thesis, Universit Paris X - Nanterre.

[27] Webb G.F., Theory of nonlinear age-dependent population dynamics. Marcel Dekker, New York, 1985. 